\title{
Influencia del capital humano en las diferencias regionales en Colombia durante el periodo 1990-2010
}

\author{
págs. 193-216 \\ Grupo de investigación: Desarrollo y Equidad \\ Línea de Investigación: Desarrollo industrial y empresarial \\ María Esperanza Cuenca Coral •
}

\section{RESUMEN}

En el periodo comprendido entre 1990 y 2010, se marca una tendencia fuerte en la influencia ejercida por la generación de capital humano, abriendo la brecha entre las diferentes regiones físicas y económicas del país. El análisis de la problemática sugiere una perspectiva modélica que va desde la estadística hasta la contabilidad, pasando por la visión y los resultados propios de la política económica. Se asume en el artículo que el análisis de tal influencia bajo los parámetros del análisis econométrico, permitirían observar, describir y explicar las diferencias regionales en el desarrollo económico del país.

Palabras clave: Capital Humano, coeficiente Gini, Región económica, Modelo Vectorial Auto-regresivo.

\begin{abstract}
Between the years 1990 and 2010, it is notable a strong tendency in the influence put for the generation of Human Capital, opening the division between the physic and economic regions in the country. The analysis of the problem suggests one modeled vision that goes from statistics to accounting, passing through the analysis and the results of the economic policy. It is assumed in the article that the analysis of that influence, under the parameters of the econometric analysis, will permit to observe, describe, and explain the regional differences in the economic development of the country.
\end{abstract}

Key Words: Human Capital, Gini Quotient, Economic Region, Auto Regressive Vectorial Model.

- Docente investigadora del programa de Economía Universidad de América. 


\section{INTRODUCCIÓN}

Este documento presenta una visión política y económica de la influencia del Capital humano en las regiones económicas de Colombia, con el fin de mostrar su incidencia en el desarrollo económico. Para el desarrollo del trabajo se tomaron algunos elementos de contabilidad de crecimiento como los propuestos por el modelo de Lucas. Además, se utilizó como metodología un vector auto-regresivo, donde las variables se hacen endógenas mediante un modelo VAR, (Modelo Vectorial Auto-regresivo) que se usa para caracterizar las interacciones simultáneas entre un grupo de variables; y consiste en un modelo de ecuaciones simultáneas formado por un sistema de ecuaciones de forma reducida sin restringir, es decir, que los valores contemporáneos de las variables no aparecen como variables explicativas; además se hará uso de un modelo data panel con el fin de llegar a establecer una comparación entre regiones y dentro de las mismas. El objetivo es analizar la incidencia del Capital Humano sobre las diferencias de Desarrollo regional en Colombia, teniendo en cuenta características y políticas económicas adoptadas en las regiones respecto al Capital Humano durante el periodo 1990-2010. Para llevarlo a cabo se hizo un de las Políticas económicas adoptadas, en lo referente al Capital Humano y a su impacto en el comportamiento económico de las regiones, seguido de un estudio de las características económicas, que permita hacer comparaciones de las regiones durante el periodo 1990-2010 y finalmente se examinaron y analizaron las características relacionadas con el capital humano de las regiones durante el periodo $1990-2010$.

\section{MÉTODO}

Se define capital humano como las habilidades y capacidades que son adquiridas mediante la educación formal, a lo que se suma la experiencia laboral, el entrenamiento y en general las relaciones en sociedad. Se hace este estudio siguiendo los lineamientos de Lucas, dentro de los cuales se busca realizar una aproximación a la contabilidad de los aportes que cada uno de los factores relacionados con el capital humano tiene sobre el desarrollo. También es importante resaltar a Weil (Destinobles, 2005), quien incorpora la salud dentro del capital humano especialmente en cuanto a la tasa de mortalidad, de acuerdo a su comportamiento, pues podría llegar a cambiar las preferencias de las personas y así funcionar como generador de capital humano.

\section{Modelo de Lucas}

Su propuesta alternativa es tomar un modelo que incluya la presencia de efectos externos, lo cual no coincide con la trayectoria del crecimiento óptimo y equilibrio competitivo. A partir de este momento, Lucas abandona el modelo de Solow y retoma el análisis de Romer, que es similar, a fin de construir un modelo que le permita obtener el óptimo y trayectoria del equilibrio de manera separada y comparados entre ellos.

\section{Teorías sobre distribución espacial y geografía económica}

La Teoría del desarrollo regional se basa en los mismos fundamentos que la teoría de desarrollo general, pues las dos intentan explicar en un sentido más amplio las condiciones y el bienestar a largo plazo de la población que se encuentra ubicada en determinado lugar. Es importante tomar en cuenta dos puntos relevantes dentro de la teoría del desarrollo regional:

- El desarrollo económico se debe a la interacción de las llamadas fuerzas centrífugas y centrípetas

- Las regiones se mueven según la actividad económica que en ella se genere. El uso que se dé a los espacios, personas y los elementos de producción, puede llegar a generar la diferencia entre un territorio y otro

Aunque no se toma como variable para el desarrollo del modelo, el PIB regional para el período 1990-2010 se incluye en el análisis, ya 
Cuenca, María Esperanza: Influencia del capital humano en las diferencias regionales en Colombia...

LÍNEA DE INVESTIGACIÓN: DESARROLLO INDUSTRIAL Y EMPRESARIAL

que es un indicador bastante pertinente que permite llegar a conclusiones útiles para el presente trabajo, pues estamos haciendo referencia al desarrollo regional, teniendo en cuenta que es una regionalización a partir de características diferentes a la proximidad geográfica. Entre los principales aspectos que se pueden resaltar respecto a este factor se encuentran:

- Región Elite representa más del 55\% del PIB total para todos los años

- Períodos más críticos por conflictos de tipo económico, como la crisis hipotecaria de 1998 en Colombia y la repercusión en Colombia de la crisis de los Estados Unidos en el año 2008

- La Región Periférica no alcanza a tener una participación de $1 \%$ o más en ninguno de los 10 períodos analizados

- Después de la Región Elite, aquella que sigue con el mayor aporte al PIB, es la Región Central, aportando alrededor del $17 \%$ al PIB

- Resto tiene casi el doble de la superficie en K2 de la Región Élite, pero las diferencias en aspectos económicos, población y condiciones sociales son abismales

Durante la elaboración de la tesis seminal de este artículo, se realizó un estudio exhaustivo de la normatividad relacionada con la salud y la educación. Sin embargo, por problemas de espacio, sólo se reseña a continuación los más importantes resultados econométricos. En los anexos se encuentran los gráficos de impulso respuesta.

\section{Modelo econométrico}

A partir de los siguientes hechos estilizados se estableció una aproximación econométrica basada en la necesidad de encontrar semejanzas y diferencias entre las regiones y dentro de las mismas; es por esto que la metodología inicial es el panel de datos (data panel) y un modelo
VAR (Vector Auto Regresivo). Las regiones que se han tomado en este trabajo son:

- Región Elite

- Región Cafetera

- Región Periférica

- Región Norte

- Región Central

Las variables que se han considerado son:

- Esperanza de vida

- Población analfabeta

- Escolaridad combinada

- Índice de logro educativo

- Índice de esperanza de vida

- Variación Porcentual del PIB

- GINI

- IDH

- PIB Regional

De las anteriores, se tomaron 5 variables finalmente para correr los modelos, estas son: Esperanza de vida, analfabetismo, GINI, IDH crecimiento. Modelo 1. Sección cruzada en 5 unidades de 90 observaciones.

Variable independiente IDH. El método ArreIlano - Bond es un modelo de data panel, estimado mediante el Método Generalizado de los momentos y permite estimar un modelo dinámico sin necesidad de instrumentos externos. Por otro lado, el argumento utilizado para obtener instrumentos de yit-1 se puede generalizar a otros regresores que no sean estrictamente exógenos. 
Tabla 1. Resultados modelo data panel regiones Colombia

\begin{tabular}{|c|c|c|c|c|c|}
\hline Variable & Coeficiente & Desv. Tip. & Estad T & Valor $\mathrm{T}$ & \\
\hline DIDH (-1) & 0,562963 & 0,0298839 & 18,838 & 0,00001 & *** \\
\hline CONST & 0,00560288 & 0,00332981 & 1,683 & 0,09244 & * \\
\hline ANAL & $2,46809 \mathrm{e}-05$ & $1,81403 \mathrm{e}-05$ & 1,361 & 0,17365 & \\
\hline EV & $-0,000126219$ & $3,48658 \mathrm{e}-05$ & $-3,620$ & 0,00029 & *** \\
\hline CREPIB & 0,000496255 & 0,000130204 & 3,811 & 0,00014 & *** \\
\hline GINI & 0,00631501 & 0,00312859 & 2,018 & 0,04354 & ** \\
\hline \multicolumn{6}{|c|}{ Suma de cuadrados de los residuos $=0,0036937$} \\
\hline \multicolumn{6}{|c|}{ Desviación típica de los residuos = 0,00663119 } \\
\hline \multicolumn{6}{|c|}{ Contraste de error es $\mathrm{AR}(1): \mathrm{z}=-1,5698$ (Valor $\mathrm{p} 0,1165)$} \\
\hline \multicolumn{6}{|c|}{ Contraste de error es $\operatorname{AR}(2): z=1,73025$ (valor $p$ 0,0836) } \\
\hline \multicolumn{6}{|c|}{ Contraste de sobreidentificación de sargan: } \\
\hline \multicolumn{6}{|c|}{ Chi - cuadrado $(78)=134,244($ valor $p 0,0001)$} \\
\hline \multicolumn{6}{|c|}{ Contraste (conjunto) de wald: } \\
\hline \multicolumn{6}{|c|}{ Chi - cuadrado $(5)=4,34055 \mathrm{E}+009($ valor $p 0,0000)$} \\
\hline
\end{tabular}

Fuente: Autor con base en Eviews 6.0

Del modelo 1 (tabla 1), se puede concluir que las variables utilizadas para explicar el Índice de Desarrollo Humano son significativas, dentro del modelo establecido, como se esperaba dentro de la perspectiva teórica. Deben tenerse en cuenta entonces el índice de desarrollo humano en un periodo inmediatamente anterior, EV, definir el crecimiento del PIB y el coeficiente GINI, resultados que podían ser predichos desde la teoría. Este análisis cruzado muestra que entre las regiones no son significativas ni la constante $\mathrm{ni}$ la variable ANAL.

Una vez corrido el VAR para cada región se arrojan los resultados que permiten llegar a conclusiones y en última instancia a realizar comparaciones entre los resultados de las diferentes regiones. Se interpretan los modelos VAR, los gráficos de Impulso respuesta y la descomposición de la varianza para cada región.

\section{VAR, función impulso respuesta y descomposición de la varianza}

Región Élite Al examinar los resultados obtenidos de manera individual, al tomar CRE como variable endógena, se observa que GINI y EV en el rezago 1 y 2 son estadísticamente significativos, con un $90 \%$ de confianza. De igual manera al analizar el valor $\mathrm{F}$ se concluye que como $|F| \geq F_{\text {c }}$ se rechaza la hipótesis nula y al rechazar la hipótesis nula de que Ho: $\beta 2=\beta 3$ $=\beta 4=0$ se dice que por lo menos uno de estos betas es diferente de cero.

Así mismo al tomar EV como variable endógena, resulta estadísticamente significativas GINI, EV y CRE en los rezagos 1 y 2 así como IDH y ANAL en el rezago 1 , con un $\alpha=0,1$, lo cual indica que ante un incremento en la esperanza de vida en la región elite el GINI disminuye y se presenta un incremento en el crecimiento económico regional. Si se analiza la prueba $F$, se concluye que como $|F| \geq F_{c r}$ se rechaza la hipótesis nula y al rechazar la hipótesis nula de que Ho: $\beta 2=\beta 3=\beta 4=0$ se dice que por lo menos uno de estos betas es diferente de cero.

Cuando la variable endógena es GINI, muestra que IDH, EV, ANAL y GINI, todas en el rezago 1 y 2 y CRE en el rezago 1 son estadísticamente significativas con un $\alpha=0,1$, lo cual indica que ante un aumento del Gini el IDH disminuye, lo cual concuerda con la teoría pues si 
Cuenca, María Esperanza: Influencia del capital humano en las diferencias regionales en Colombia...

LÍNEA DE INVESTIGACIÓN: DESARROLLO INDUSTRIAL Y EMPRESARIAL

el nivel de desigualad se incrementa el desarrollo disminuye. De igual manera al analizar el valor F se concluye que como $|F| \geq F_{c r} \mid$ se rechaza la hipótesis nula y al rechazar la hipótesis nula de que Ho: $\beta 2=\beta 3=\beta 4=0$ se dice que por lo menos uno de estos betas es diferente de cero.

En relación al IDH como variable endógena se observa que GINI en el rezago 1 es estadísticamente significativa, con un nivel de confianza del $90 \%$, mostrando que para el desarrollo de la región Elite hay una relación inversa con el coeficiente de Gini, es decir, a mayor nivel de desarrollo menor desigualdad. De igual forma al analizar el valor $F$ se concluye que como $|F| \geq F_{c r}$ se rechaza la hipótesis nula y al rechazar la hipótesis nula de que Ho: $\beta 2=\beta 3=$ $\beta 4=0$ se dice que por lo menos uno de estos betas es diferente de cero.

Se arrojan también los resultados de la regresión para cada ecuación, computando los resultados por separado. Así en la ecuación que define al IDH como variable dependiente se alcanza una bondad del ajuste del 0,987, es decir que $98 \%$ de las diferencias respecto a la media quedan explicadas por la función, lo cual significa que para la región Elite el coeficiente de Gini, la tasa de analfabetismo, la esperanza de vida y el crecimiento económico representan el $98 \%$ del desarrollo de la región.

Las respuestas de IDH con respecto a EV, ANAL, CRE y GINI son las esperadas. Así mismo, ante un aumento de GINI la tasa de analfabetismo aumenta y el PIB disminuye. Esta relación se explica pues al incrementarse la desigualdad, indica que los ingresos están concentrados en una parte de la población, por lo cual el nivel de analfabetismo crece.

- ANAL: Sí misma, esperanza de vida y crecimiento

- IDH: Tasa analfabetismo, crecimiento económico y esperanza de vida

- CRECIMIENTO: Sí misma, analfabetismo y esperanza de vida
- EV: Crecimiento, esperanza de vida y analfabetismo

- GINI: Analfabetismo, esperanza de vida y crecimiento

\section{Región Norte}

Al analizar los resultados obtenidos de la primera columna que toma como variable endógena IDH, se observa que la esperanza de vida y el IDH rezagadas un periodo, es significativa $\operatorname{con} \alpha=$ 0,1 lo cual quiere decir que, con un $90 \%$ de confianza se afirma que el desarrollo de la región norte depende principalmente de la esperanza de vida de su población. Es decir a mayor esperanza de vida mayor desarrollo.

Tomando como variable endógena el GINI, resultan significativas con un $\alpha=0,05$ la tasa de analfabetismo y la esperanza de vida rezagadas un periodo, es decir que con un $95 \%$ de confianza se puede afirmar el índice de Gini de la región norte se explica principalmente por la tasa de analfabetismo y la esperanza de vida. Por lo cual a mayor analfabetismo y menor esperanza de vida se presenta un incremento en el nivel de concentración del ingreso.

Tomando como variable endógena EV, muestra que GINI y ANAL rezagadas un periodo son significativas con un nivel de confianza del $90 \%$, lo cual quiere decir que al presentarse una disminución en la concentración del ingreso y de la tasa de analfabetismo la esperanza de vida de la región norte se incrementa.

Si se toma como variable endógena el CRE, las variables estadísticamente significativas con un $\alpha=0,1$ son GINI y EV rezagadas un periodo. De igual manera en la quinta columna la variable dependiente es ANAL, cuyo comportamiento depende de EV $(-1)$, pues a mayor esperanza de vida menor tasa de analfabetismo.

Así mismo al analizar el valor $\mathrm{F}$ para cada variable se concluye que como $|F| \geq F_{c r} \mid$ se rechaza la hipótesis nula y al rechazar la hipótesis 
nula de que Ho: $\beta 2=\beta 3=\beta 4=0$ se dice que por lo menos uno de estos betas es diferente de cero. En la ecuación la que define al IDH como variable dependiente, se alcanza una bondad del ajuste del 0,975 , es decir que para la región norte la tasa de crecimiento del PIB (CRE), la esperanza de vida (EV), el Índice de Desarrollo Humano (IDH), el índice de Gini (GINI) y tasa de analfabetismo (ANAL), representan el $98 \%$ del desarrollo de esa región.

Las respuestas de IDH con respecto a EV, ANAL, CRE y GINI son las esperadas, pues ante un incremento en el nivel de desarrollo de la región norte se observa un aumento en la esperanza de vida y el crecimiento económico y una disminución de la tasa de analfabetismo y la desigualdad en la distribución de la riqueza. Así mismo, ante un aumento del crecimiento económico el nivel de desarrollo presenta el mismo comportamiento, la esperanza de vida aumenta y el analfabetismo disminuye.

- IDH: Sí mismo, esperanza de vida, crecimiento económico y GINI.

- GINI: Sí misma, IDH y esperanza de vida y crecimiento

- EV: Gini, IDH, esperanza de vida y crecimiento

- CRE: Esperanza de vida, GINI, crecimiento e IDH.

- ANAL: Gini, IDH, crecimiento económico y esperanza de vida.

\section{Región Periférica}

Al analizar los resultados obtenidos, cuando se toma como variable endógena CRE, se observa que esta con un nivel de significancia de $\alpha=0,1$, depende de IDH, GINI y EV rezagadas todas uno y dos periodos y de CRE y ANAL rezagadas un periodo, es decir que el crecimiento económico de la región periférica depende en gran medida del mejoramiento de la calidad de vida de la población.

Si se toma como variable endógena el ANAL, resultan significativas IDH (-2), GINI (-2) y ANAL (-1). Por lo cual el comportamiento de la tasa de analfabetismos en la región periférica depende de la igualdad o desigualdad que exista en la distribución de la riqueza así como de la calidad de vida de la población, pues a mayor desigualdad y baja calidad de vida aumenta la tasa de analfabetismo.

Cuando se toma como variable endógena EV, observándose que GIN I (-1), EV (-1), ANAL $(-1)$ y CRE (-2), son significativas con $\alpha=0,1$ lo cual quiere decir que, con un $90 \%$ de confianza se afirma que la esperanza de vida en la región periférica depende negativamente de GINI y ANAL y positivamente de CRE. Si la variable dependiente es GINI, esta depende positivamente de la tasa de analfabetismo rezagada un periodo, con un nivel de confianza del $95 \%$, por lo cual ante un incremento en la desigualdad de los ingresos aumenta el porcentaje de la población que no sabe leer ni escribir.

Cuando se toma como variable endógena IDH, se evidencia que CRE (-1), GINI (-1), ANAL (-2) y EV (-1) (-2), son estadísticamente significativos con $\alpha=0,5$, lo cual se ajusta al planteamiento teórico el cual afirma que ante un incremento de la esperanza de vida y del crecimiento económico y una disminución de la tasa de analfabetismo y de la desigualdad del ingreso el desarrollo aumenta.

Así mismo al analizar el valor $\mathrm{F}$ para cada variable se concluye que como $|F| \geq F_{c r} \mid$ se rechaza la hipótesis nula y al rechazar la hipótesis nula de que Ho: $\beta 2=\beta 3=\beta 4=0$ se dice que por lo menos uno de estos betas es diferente de cero. En la ecuación que define al IDH como variable dependiente, se alcanza una bondad del ajuste del 0,99, es decir que $99 \%$ de las diferencias respecto a la media quedan explicadas por la función, lo cual significa que para la región Periférica el coeficiente de Gini, la tasa de anal- 
Cuenca, María Esperanza: Influencia del capital humano en las diferencias regionales en Colombia...

LÍNEA DE INVESTIGACIÓN: DESARROLLO INDUSTRIAL Y EMPRESARIAL

fabetismo, la esperanza de vida y el crecimiento económico representan el 99\% del desarrollo de la región.

\section{Región Central}

Al analizar los resultados obtenidos, se observa que tomando ANAL como variable endógena, con un nivel confianza del $90 \%$, está explicada por EV(-1), IDH(-1), GINI(-1)(-2) y ANAL(-1)(-2), Es decir que la tasa de analfabetismo de hoy depende del comportamiento de la esperanza de vida y del Índice de Desarrollo Humano presentado en el periodo anterior y del coeficiente de Gini uno y dos periodos anteriores. De igual manera la analizar EV como variable dependiente, resulta estadísticamente significativo con un $\alpha=0,05$, ANAL $(-1)$, por lo cual ante un incremento de la tasa de analfabetismo se presenta una disminución de la esperanza de vida de la población de la región central, pues al no saber leer ni escribir, el acceso al mercado laboral es difícil, generando que el ingreso sea bajo, lo cual afecta la calidad de vida y así su EV.

Al tomar a IDH como variable endógena, se observa que IDH (-1) es significativa con un nivel de confianza del $95 \%$, es decir que el desarrollo de la región central de hoy depende del presentado en el periodo anterior.

Así mismo al analizar el valor $\mathrm{F}$ para cada variable se concluye que como $|F| \geq F_{c r} \mid$ se rechaza la hipótesis nula y al rechazar la hipótesis nula de que Ho: $\beta 2=\beta 3=\beta 4=0$ se dice que por lo menos uno de estos betas es diferente de cero. En la cuarta ecuación en la que define al IDH como variable dependiente se alcanza una bondad del ajuste del 0,987, es decir que para la región central el coeficiente de Gini, la tasa de analfabetismo, la esperanza de vida y el crecimiento económico representan el 99\% del desarrollo de la región.

Las respuestas de IDH con respecto a CRE y GINI son las esperadas, pues ante un incremento en el crecimiento económico y una disminución del coeficiente de Gini el desarrollo de la región se incrementa. Así mismo, ante un aumento de CRE la tasa de analfabetismo disminuye y la esperanza de vida aumenta. Esta relación se explica pues al presentarse crecimiento económico, indica que la región tiene mayores ingresos, lo cual genera mayor inversión en pos del mejoramiento de la calidad de vida de la población, por cual se incrementa la esperanza de vida y se disminuye el analfabetismo.

- ANAL: Sí misma, IDH, Gini y esperanza de vida

- GINI: Analfabetismo, Esperanza de vida e IDH

- EV: Sí misma, analfabetismo, IDH y Gini

- IDH: Sí misma, crecimiento y analfabetismo

- CRE: Esperanza de vida y analfabetismo

\section{Región Cafetera}

Al analizar los resultados obtenidos, se observa primero ANAL como variable endógena, la cual con un nivel confianza del 95\%, esta explicada por la esperanza de vida rezagada dos periodos, es decir que la tasa de analfabetismo de la región cafetera depende negativamente de $\mathrm{EV}$, por lo cual al incrementarse la esperanza de vida el porcentaje de población que no sabe leer ni escribir disminuye. Cuando se muestra como variable dependiente a CRE, la cual con un $90 \%$ de confianza depende negativamente del GINI (-2) y positivamente del IDH (-1) y CRE (-1). Es decir que a menor desigualdad en el ingreso y mayor desarrollo se presenta un incremento en el crecimiento económico. Con base en lo anterior una disminución del nivel de desigualdad tenderá a aumentar la productividad media de la inversión, permitiendo que los individuos de menores ingresos realicen inversiones que ofrecen tasas de rendimiento relativamente altas, incrementando así el crecimiento económico (Fossati, 2002). 
Tomando como variable endógena EV, la cual con un $90 \%$ de confianza depende de GINI (-1) (-2), IDH (-1), ANAL (-2) y EV (-2). Lo anterior indica que a medida que exista igual en los ingresos las personas son más sanas, porque poseen mayores bienes y servicios que ayudan a tener buena salud y así mayor esperanza de vida; de igual manera el desarrollo es un ítem importante al momento de analizar la esperanza de vida pues este permite dimensionar la calidad de vida de la población la cual se refleja en EV.

Si se toma como variable endógena el GINI, resulta estadísticamente significativa EV (-1) (-2), con un $90 \%$ de confianza, es decir que a mayor desigualdad en el ingreso menor es la esperanza de vida de la población de la región cafetera.

Cuando IDH es la variable dependiente, se observa que GINI (-2), EV (-2) e IDH (-1) (-2) son significativas con un $\alpha=0,1$, es decir entre menor sea la calidad de vida de la población, reflejada en mayor desigualdad en el ingreso y deterioro en la salud el desarrollo de la región disminuye.

En la ecuación que define al IDH como variable dependiente se alcanza una bondad del ajuste del 0,992, es decir que para la región central el coeficiente de Gini, la tasa de analfabetismo, la esperanza de vida y el crecimiento económico representan el $99 \%$ del desarrollo de la región.

Las respuestas de CRE con respecto a EV, ANAL, IDH y GINI son las esperadas. Así mismo, ante un aumento de ANAL tanto el crecimiento como el IDH disminuyen. Esta relación se explica pues al incrementarse el porcentaje de la población que no saben leer ni escribir indica que la cualificación del recurso humano es baja generando como resultado menos habilidades, baja productividad y menor capacidad de los trabajadores para mejorar las condiciones de vida.

- ANAL: Sí misma y Gini

- CRE: Sí misma, analfabetismo y Gini
- EV: Sí misma y analfabetismo

- GINI: Sí misma, esperanza de vida y crecimiento

- IDH: Esperanza de vida, analfabetismo y crecimiento

\section{CONCLUSIONES Y RECOMENDACIONES}

Uno de los puntos que se analizaron en el presente trabajo es el referente a las políticas económicas adoptadas en cuanto a capital humano (salud y educación) y su impacto en el comportamiento de las regiones. En Colombia, se han aplicado diversas políticas en estos aspectos durante el período 1990-2010, las cuales suelen tener alguna variación de un gobierno a otro, pero en general, estas se mantienen y se tienden a mejorar; por ejemplo, haciendo referencia a la educación, dos temas que siempre se han mantenido y se traducen en forma de políticas son la cobertura y la calidad; que desde 1992 se ha velado por mejorarla y actualmente es una de las metas del Ministerio de Educación junto con el Gobierno Nacional.

Se consideró importante también, tocar un poco las políticas en materia de empleo, ya que existe una estrecha relación entre las condiciones de salud de una persona, su educación y la calidad de empleo que logre conseguir y por ende el nivel de sus ingresos, encontrando que en Colombia hay una serie de políticas sectoriales y estrategias para combatir el desempleo, mejorar las condiciones laborales y crear empleo para las personas más vulnerables.

Durante la década de los 90 se formularon una serie de políticas en materia de salud y educación, algunas de ellas determinaron el comportamiento del capital humano en relación con el desarrollo de cada región, pero es importante tener en cuenta que dicho comportamiento depende también de programas de educación, como la creación y ampliación de planteles edu- 
Cuenca, María Esperanza: Influencia del capital humano en las diferencias regionales en Colombia...

LÍNEA DE INVESTIGACIÓN: DESARROLLO INDUSTRIAL Y EMPRESARIAL

cativos y de hospitales y planes en cuanto a la salud. Para cada región, se dio lugar a un análisis que permitiera identificar la o las variables que mayor incidía en cada una.

Los resultados del VAR para la Región Norte muestran que con un $90 \%$ de confianza se afirma que el desarrollo en dicha región depende principalmente de la esperanza de vida de su población. Es decir a mayor esperanza de vida mayor desarrollo. En cuanto a la Región Periférica el crecimiento económico depende en gran medida del mejoramiento de la calidad de vida de la población, es decir, tanto del desarrollo humano como del analfabetismo. En el VAR de la Región Central se observa que la tasa de analfabetismo de hoy depende del comportamiento de la esperanza de vida y del Índice de Desarrollo Humano presentado en el periodo anterior y del coeficiente de GINI uno y dos periodos anteriores. Así mismo ante un incremento en la esperanza de vida en la Región Elite el GINI disminuye y se presenta un incremento en el crecimiento económico regional. Por ultimo en la Región Cafetera la tasa de analfabetismo depende negativamente de EV, por lo cual al incrementarse la esperanza de vida el porcentaje de población que no sabe leer ni escribir disminuye.

El estudio permitió hacer comparaciones entre las regiones y al interior de las mismas; teniendo en cuenta que la regionalización que se tomó da como resultado 5 regiones: Región Elite, Región Cafetera, Región Periférica, Región Norte y Región Central. Se resaltan las características más importantes para cada una de ellas, tales como el PIB Regional, variable que además de incluirse en el desarrollo del modelo, valió la pena analizar de forma independiente, pues es una muestra clara del comportamiento de cada región en materia económica y su aporte al PIB y por otro lado, pero no menos importante, permite ver la evolución a precios constantes de dicha variable, ya que en este capítulo se encuentra también una tabla (Tabla 1) que indica el comportamiento del PIB y del PIB per cápita para el año 1998, de manera que se buscaba analizar datos más actualizados y que abarque el período de estudio del presente trabajo. Respecto a lo anterior, se encuentra que efectivamente la Región Elite es la que mayor desarrollo tiene y por el contrario la Periférica carece de más recursos y bienestar para su población, debido a que los departamentos que la conforman presentan altos índices de analfabetismo y baja esperanza de vida, fenómenos que se ven reflejados en las oportunidades laborales con las que cuentan y asimismo los ingresos que estas les generan; razón por la cual su aporte al PIB total Nacional no alcanza a ser del $1 \%$.

Una conclusión muy importante que se desprende de este análisis, es que el PIB regional permite corroborar en qué proporción se ha visto afectada cada región y el país en general frente a sucesos como las crisis que se han presentado en algunas ocasiones, tales como en 1998 en Colombia y 2007 en Estados Unidos, que tuvo por supuesto un efecto directo en el país, por la gran dependencia que tiene del país norteamericano.

Vale la pena resaltar una conclusión general pero muy importante en cuanto al desarrollo del modelo econométrico, se trata de la gran influencia del coeficiente GINI dentro del desarrollo de cada región, pues aunque se trabajaron 4 variables más, se encontró con que el GINI es la variable común que más incide en las 5 regiones, esto evidencia efectivamente el comportamiento de este indicador en Latinoamérica y específicamente en Colombia, país que presenta uno de los más altos índices, es decir, existe una alta desigualdad en la distribución de los ingresos, evidentemente sesgada hacia las regiones con mejor comportamiento económico.

Con el presente trabajo se logra evidenciar que se llega a un modelo de desarrollo que beneficia o privilegia en gran medida a la parte empresarial, pues son las regiones con mayores recursos las que presentan mayor desarrollo, esto es muy importante para el crecimiento de un país, pues incentiva e impulsa su actividad económica, pero vale la pena mencionar que 
esto hace que se descuide la parte social, que viene enmarcando en sí el capital humano y este fenómeno puede traer implicaciones en el largo plazo para cada región y para el país.

Se recomienda para futuros trabajos, realizar un estudio similar pero al interior de cada Departamento que conforma la región, ya que de esta forma se puede tener un resultado un poco más detallado del comportamiento de cada departamento, donde se puede encontrar además el impacto del capital humano de cada una de ellos a su región, al país y a sí mismos.

Teniendo en cuenta que el comportamiento de cada región es diferente y asimismo las variables que son más determinantes para el desarrollo de cada una, surge la necesidad de establecer políticas diferenciales para cada región y cada variable.

\section{REFERENCIAS}

ASIECO (Asociación lberoamericana de Educación Combinada) Documento en línea. Disponible en http://www. asieco.org/home/about

Barrera, F.; Domínguez, C. "Educación Básica en Colombia: Opciones futuras de política. DNP. 2006." Documento en línea. Disponible en https:// www.dnp.gov.co/Portals/0/ archivos/documentos/DDS/ Pobreza/En_Que_Vamos/ EDUCACION_BASICA.pdf

Barón, J. "Las Regiones Económicas de Colombia: Un análisis de Clústeres" Banco de la República. Cartagena de Indias, Enero de 2002. Documento en línea. Disponible en < http://www.banrep.gov. co/documentos/publicaciones/ pdf/DTSER23-REGIONESECONOMICAS.pdf>

Bustamante, J.; Jaramillo, C. Cuentas departamentales - base 2005 Años 2008 definitivo, 2009 provisional y 2010 preliminar. Bogotá 2011.
Cuenca, M.; Penagos, I. "Una aproximación empírica fundamentada en la perspectiva capital humano (1960 2009)". Marzo 2012.

DANE. (Departamento Administrativo Nacional de Estadística). "Definiciones". Documento en línea, disponible en <http://www.google.com.co/ url?sa=t\&rct=j\&q=definicion $\% 20$ esperanza $\% 20$ de $\% 20$ vida $\% 20$ dane \& source $=$ web\& $c d=5 \&$ ved $=0 C E I Q F j A E \&$ url=http $\% 3 \mathrm{~A} \% 2$ $\mathrm{F} \% 2 \mathrm{Fwww}$.dane.gov.co $\% 2 \mathrm{Fce}$ nso $\% 2$ Ffiles $\% 2 F$ presentacion es $\% 2 \mathrm{FIndidemograficosDptos.}$ xls\&ei=QG5DUdubE4T

E4AOf-IGACA\&usg=AFQj CNHyAhuvyehsYW_VIS_nnEAxg_ $d Z 3 w \& b v m=b v .43828540, d$. eWU>

Del Sol, J. "Resumen de Desarrollo y Libertad de Amartya Sen". Junio de 2004. Documento en línea. Disponible en Internet: < http://makgregory.blogspirit.com/ files/desarrolloylibertad_josegregoriodelsolcobos.pdf

Destinobles, A. El modelo de Mankiw, Romer y Weil (1992) en el programa de investigación neoclásico. 2005. Revista Aportes, Vol. 10, No. 30. Universidad Autónoma de Puebla. Puebla, México.

DNP. (Departamento Nacional de Planeación) "Política de Empleo" Gobierno Nacional. Seminario de Empleo y Economía. Documento en línea, disponible en < http:// www.banrep.gov.co/documentos/seminarios/pdf/presen_ echeverry.pdf>

Fossati, V. Desigualdad y Crecimiento. Un Análisis para las Provincias

Argentinas. Documento de Trabajo Nro. 43. Septiembre 2002, Buenos Aires, Argentina.

Huayamave, X. "Crecimiento económico" Documento en línea. Disponible en Internet http://www.monografias.com/trabajos14/crecimientoecon/crecimientoecon. shtmI\#DESARR

Instituto de Estudios e Investigaciones de Trabajo (Cuba). "Teoría del Capital Humano" Documento en línea. Disponible en internet: http:// 
Cuenca, María Esperanza: Influencia del capital humano en las diferencias regionales en Colombia...

LÍNEA DE INVESTIGACIÓN: DESARROLLO INDUSTRIAL Y EMPRESARIAL

es.scribd.com/doc/17419558/ TEORIA-DEL-CAPITAL-HUMANO

Ken y Chan Ceh. "Clústeres: una alternativa para el desarrollo regional de pequeñas economías" en Contribuciones a la Economía, abril 2008 Documento en línea. Disponible en internet, en línea <http:// www.eumed.net/ce/2008a/

Lozano, R. "Modelos de crecimiento endógeno: externalidades del capital humano" Documento en línea. Disponible en Internet <http://www. eumed.net/eve/resum/06-02/ rlc.htm
Novales, A. "Modelos Vectoriales Auto-regresivos VAR". Septiembre 2011. Documento en línea, disponible en http:// www.ucm.es/info/ecocuan/ anc/ectriaqf/VAR.pdf

Ospina, N. "Una nueva visión del capital humano: evidencia para Colombia" XVI Jornadas de la Asociación de Economía de la Educación.

Palacio, D. "Plan de Salud Pública 2007-2010". Ministerio de la Protección Social http://www2.paho.org/ hq/dmdocuments/2010/Politicas_Nacionales_Salud-Colombia_2007-2010.pdf
PNUD. "Índice de desarrollo humano. Documento en línea" Disponible en <http://www.pnud.org.co/ sitio.shtml?apc=i1---67019$\& s=a \& m=a \& e=B \& c=02008 \#$. UUOShRdyFd0>

Zuleta, J; López, L. y Sánchez, D. "Evolución de las disparidades económicas espaciales en Colombia: un análisis regional". Marzo 2008. Documento en línea. Disponible en Internet, en línea $<$ http://institutodeestudiosurbanos.info/dmdocuments/ Mesa1_5_EvoluciondelasdisparidadeseconomicasespacialesenColombia.pdf 


\section{ANEXOS}

Tabla 2.

\begin{tabular}{|c|c|c|c|c|c|}
\hline & ANAL & CRE & EV & GINI & IDH \\
\hline \multirow{3}{*}{ ANAL(-1) } & -0.370943 & 2.312200 & 0.083273 & -0.011874 & -0.006310 \\
\hline & $(0.59058)$ & $(4.59734)$ & $(0.03597)$ & $(0.00365)$ & $(0.00816)$ \\
\hline & {$[-0.62810]$} & [0.50294] & [2.31485] & [-3.25193] & [-0.77292] \\
\hline \multirow{3}{*}{ ANAL(-2) } & -0.289938 & -1.671676 & -0.013370 & 0.006666 & -0.011271 \\
\hline & $(0.62616)$ & $(4.87430)$ & $(0.03814)$ & $(0.00387)$ & $(0.00866)$ \\
\hline & {$[-0.46304]$} & {$[-0.34296]$} & {$[-0.35055]$} & [1.72191] & {$[-1.30220]$} \\
\hline \multirow{3}{*}{ CRE(-1) } & -0.020266 & 0.840620 & -0.005584 & 0.000199 & 0.000953 \\
\hline & $(0.03737)$ & $(0.29090)$ & $(0.00228)$ & $(0.00023)$ & $(0.00052)$ \\
\hline & {$[-0.54231]$} & [2.88972] & {$[-2.45298]$} & [0.86021] & [1.84499] \\
\hline \multirow{3}{*}{ CRE(-2) } & -0.012687 & 0.025633 & 0.008426 & 0.00995 & 0.000805 \\
\hline & $(0.03311)$ & $(0.25771)$ & $(0.00202)$ & $(0.00020)$ & $(0.00046)$ \\
\hline & {$[-0.38324]$} & [0.09946] & [4.17836] & [4.86208] & [1.75914] \\
\hline \multirow{3}{*}{$\mathrm{EV}(-1)$} & 0.857326 & 56.26629 & 0.435176 & 0.111970 & -0.013803 \\
\hline & (3.10232) & $(24.1499)$ & $(0.18897)$ & $(0.01918)$ & $(0.04288)$ \\
\hline & {$[0.27635]$} & [2.32988] & [2.30289] & [5.83782] & {$[-0.32187]$} \\
\hline \multirow{3}{*}{$\mathrm{EV}(-2)$} & -2.042128 & -54.19437 & 0.656124 & -0.088345 & 0.012352 \\
\hline & (3.37599) & (26.2803) & $(0.20564)$ & $(0.02087)$ & $(0.04667)$ \\
\hline & {$[0.60490]$} & {$[-2.06217]$} & [3.19065] & {$[-4.23270]$} & {$[0.26470]$} \\
\hline \multirow{3}{*}{ GINI(-1) } & 4.821162 & -190.1402 & -1.305685 & -0.513983 & -0.389136 \\
\hline & $(10.7373)$ & (83.5838) & $(0.65403)$ & $(0.06638)$ & $(0.14842)$ \\
\hline & [0.44901] & [-2.38252] & {$[-1.99636]$} & {$[-7.74270]$} & {$[-2.62184]$} \\
\hline \multirow{3}{*}{ GINI(-2) } & -2.983468 & 230.9085 & -2.349484 & -0.862997 & 0.030205 \\
\hline & (15.5628) & (121.148) & $(0.94797)$ & $(0.09622)$ & $(0.21512)$ \\
\hline & {$[-0.19171]$} & [1.90600] & {$[-2.47844]$} & {$[-8.96929]$} & {$[0.14041]$} \\
\hline \multirow{3}{*}{$\mathrm{IDH}(-1)$} & -24.02572 & -220.5933 & 3.251926 & -2.047267 & 0.360777 \\
\hline & (34.8675) & $(271.425)$ & $(2.12386)$ & $(0.21557)$ & $(0.48197)$ \\
\hline & {$[-0.68906]$} & {$[-0.81272]$} & [1.53114] & {$[-9.49710]$} & {$[0.74854]$} \\
\hline \multirow{3}{*}{$\mathrm{IDH}(-2)$} & 4.089175 & 60.83146 & -3.060511 & 1.605975 & -0.327387 \\
\hline & (17.1981) & $(133.878)$ & $(1.04758)$ & $(0.10633)$ & $(0.23773)$ \\
\hline & {$[0.23777]$} & {$[0.45438]$} & {$[-2.92151]$} & [15.1041] & {$[-1.37715]$} \\
\hline \multirow{3}{*}{ C } & 106.2662 & -57.91143 & -4.496405 & -0.078232 & 1.148414 \\
\hline & $(66.4696)$ & (517.431) & $(4.04883)$ & $(0.41095)$ & $(0.91881)$ \\
\hline & [1.59872] & {$[-0.11192]$} & {$[-1.11054]$} & {$[-0.19037]$} & [1.24990] \\
\hline R-squared & 0.986044 & 0.852381 & 0.999962 & 0.996943 & 0.987530 \\
\hline Adj. R-squared & 0.962784 & 0.606350 & 0.999899 & 0.991848 & 0.966747 \\
\hline Sum sq. resids & 0.509310 & 30.86315 & 0.001890 & 1.95E-05 & 9.7E-05 \\
\hline S.E. equation & 0.291350 & 2.268007 & 0.017747 & 0.001801 & 0.004027 \\
\hline F-statistic & 42.39183 & 3.464522 & 15795.21 & 195.6763 & 47.51570 \\
\hline Log likelihood & 5.695298 & -29.19093 & 53.26669 & 92.15788 & 78.47950 \\
\hline Akaike AIC & 0.624083 & 4.728344 & -4.972551 & -9.547986 & -7.938764 \\
\hline Schwarz SC & 1.163221 & 5.267482 & -4.433413 & -9.008848 & -7.399626 \\
\hline Mean dependent & 4.157362 & 3.433614 & 71.88312 & 0.526083 & 0.801184 \\
\hline S.D. dependent & 1.510248 & 3.614841 & 1.763327 & 0.019950 & 0.022085 \\
\hline
\end{tabular}


Cuenca, María Esperanza: Influencia del capital humano en las diferencias regionales en Colombia...

LÍNEA DE INVESTIGACIÓN: DESARROLLO INDUSTRIAL Y EMPRESARIAL

Tabla 3.

\begin{tabular}{|c|c|c|c|c|c|}
\hline & IHD & GINI & EV & CRE & ANAL \\
\hline \multirow{3}{*}{$\mathrm{IDH}(-1)$} & 1.098863 & -0.009144 & 2.984866 & 90.90453 & -47.89071 \\
\hline & $(0.36493)$ & $(0.56940)$ & $(4.02742)$ & (205.489) & $(27.3141)$ \\
\hline & [3.01118] & {$[-0.01606]$} & {$[0.74114]$} & {$[0.44238]$} & {$[-1.75333]$} \\
\hline \multirow{3}{*}{$\mathrm{IDH}(2)$} & -0.911496 & 0.344470 & -12.32648 & -184.0085 & 60.95336 \\
\hline & $(0.34826)$ & $(0.54339)$ & $(3.84346)$ & (196.103) & $(26.0665)$ \\
\hline & {$[-2.61729]$} & [0.63393] & {$[-3.20713]$} & {$[-0.93833]$} & [2.33838] \\
\hline \multirow{3}{*}{ GINI(-1) } & 0.329703 & -0299166 & -0.804196 & 92.45085 & -29.40102 \\
\hline & $(0.19722)$ & $(0.30772)$ & $(2.17656)$ & $(111.054)$ & $(14.7615)$ \\
\hline & {$[1.67175]$} & {$[-0.97219]$} & {$[-0.36948]$} & [0.83249] & {$[-1.991731]$} \\
\hline \multirow{3}{*}{ GINI(2) } & -0.020143 & -0.382015 & -5.377237 & -80.90783 & 20.07534 \\
\hline & $(0.14749)$ & $(0.23013)$ & $(1.62771)$ & (83.0498) & $(11.0392)$ \\
\hline & {$[-0.13657]$} & {$[-1.66002]$} & {$[-3.30356]$} & {$[-0.97421]$} & [1.81855] \\
\hline \multirow{3}{*}{$E V(-1)$} & -0.000902 & -0.018636 & -0.039684 & 2.934123 & -0.333619 \\
\hline & $(0.01117)$ & $(0.01744)$ & $(0.12333)$ & $(6.29257)$ & $(0.83642)$ \\
\hline & {$[-0.08072]$} & {$[-1.06878]$} & {$[-0.32177]$} & [0.46628] & {$[-0.39886]$} \\
\hline \multirow{3}{*}{$\mathrm{EV}(2)$} & 0.031104 & -0.002343 & 0.145528 & -2.020237 & -2.898294 \\
\hline & $(0.01331)$ & $(0.02076)$ & $(0.14686)$ & (7.49296) & $(0.99598)$ \\
\hline & [2.33745] & {$[-0.11285]$} & {$[0.99096]$} & {$[-0.26962]$} & {$[-2.90999]$} \\
\hline \multirow{3}{*}{ CRE $(-1)$} & 0.000189 & $-1.52 e-05$ & 0.003927 & 0.225358 & 0.018931 \\
\hline & $(0.00059)$ & $(0.00093)$ & $(0.00656)$ & $(0.33460)$ & $(0.04448)$ \\
\hline & [0.31889] & {$[-0.01642]$} & [0.59887] & [0.67352] & [0.42564] \\
\hline \multirow{3}{*}{ CRE(2) } & 0.000787 & 0.000523 & 0.006543 & 0.145512 & -0.048134 \\
\hline & $(0.00060)$ & $(0.00093)$ & $(0.00658)$ & $(0.33565)$ & $(0.04462)$ \\
\hline & [1.31948] & {$[0.56214]$} & {$[0.99463]$} & {$[0.43352]$} & {$[-1.07885]$} \\
\hline \multirow{3}{*}{ ANAL(-1) } & 0.007623 & 0.010905 & -0.077154 & -0.275409 & -0.550156 \\
\hline & $(0.00367)$ & $(0.00573)$ & $(0.04051)$ & $(2.06692)$ & $(0.27474)$ \\
\hline & {$[2.07680]$} & {$[1.90403]$} & {$[-1.90458]$} & {$[0.13325]$} & {$[-2.00246]$} \\
\hline \multirow{3}{*}{ ANAL(-2) } & -0.007329 & 0.008434 & -0.172381 & -0.235485 & 0.744437 \\
\hline & $(0.00410)$ & $(0.00640)$ & $(0.04525)$ & (2.30853) & $(0.30686)$ \\
\hline & {$[-1.78764]$} & [1.31843] & [-3.80992] & {$[-0.10201]$} & {$[2.42602]$} \\
\hline \multirow{3}{*}{ C } & -1.742984 & 1.845085 & 79.06963 & 6.392149 & 239.9455 \\
\hline & $(0.94381)$ & (1.47263) & $(10.4161)$ & $(531.457)$ & $(70.6425)$ \\
\hline & {$[-1.84675]$} & [1.25292] & [7.59109] & {$[0.01203]$} & [3.39662] \\
\hline R-squared & 0.975499 & 0.987081 & 0.998578 & 0.501659 & 0.994234 \\
\hline Adj. R-squared & 0.940497 & 0.968624 & 0.996547 & -0.210256 & 0.985997 \\
\hline Sum sq. resids & 0.000258 & 0.000627 & 0.031375 & 81.67759 & 1.443110 \\
\hline S.E. equation & 0.06066 & 0.009465 & 0.066948 & 3.415879 & 0.454047 \\
\hline F-statistic & 27.87018 & 53.48194 & 491.6900 & 0.704661 & 120.7041 \\
\hline Log likelihood & 74.84951 & 66.84169 & 31.62825 & -39.15257 & -2.828749 \\
\hline Akaike AIC & -7.094390 & -6.204632 & -2.292028 & 5.572507 & 1.536528 \\
\hline Schwarz SC & -6.550274 & -5.660516 & -1.747912 & 6.116623 & 2.080644 \\
\hline Mean dependent & 0.755301 & 0.492575 & 73.16066 & 3.944118 & 13.04082 \\
\hline S.D. dependent & 0.024869 & 0.053436 & 1.139385 & 3.105016 & 3.837010 \\
\hline
\end{tabular}


Tabla 4.

\begin{tabular}{|c|c|c|c|c|c|}
\hline & CRE & ANAL & EV & GINI & IDH \\
\hline \multirow{3}{*}{ CRE $(-1)$} & 0.27813 & 0.002857 & 0.003598 & 0.000277 & 0.000765 \\
\hline & $(0.21385)$ & $(0.00952)$ & $(0.00252)$ & $(0.00050)$ & $(0.00024)$ \\
\hline & [1.26636] & {$[0.30018]$} & [1.42911] & {$[0.54895]$} & [3.14723] \\
\hline \multirow{3}{*}{ CRE(-2) } & -0.285182 & -0.000442 & 0.006620 & -0.000401 & 0.000230 \\
\hline & $(0.14086)$ & $(0.00627)$ & $(0.00166)$ & $(0.00033)$ & $(0.00016)$ \\
\hline & {$[-2.02453]$} & {$[-0.07045]$} & [3.99197] & {$[-1.20785]$} & {$[1.43759]$} \\
\hline \multirow{3}{*}{ ANAL(-1) } & 2.232835 & 0.357943 & -0.120067 & 0.020639 & -0.004555 \\
\hline & $(3.41714)$ & $(0.15208)$ & $(0.04023)$ & $(0.00806)$ & $(0.00388)$ \\
\hline & {$[0.65342]$} & [2.35372] & {$[-2.98470]$} & {$[-2.56183]$} & {$[-1.17331]$} \\
\hline \multirow{3}{*}{ ANA(-2) } & 18.62701 & -0.224177 & -0.018481 & 0.026945 & -0.030936 \\
\hline & $(7.94771)$ & $(0.35370)$ & $(0.09356)$ & $(0.01874)$ & $(0.00903)$ \\
\hline & [2-34370] & {$[-0.63380]$} & {$[-0.19753]$} & [1.43799] & [3.4218] \\
\hline \multirow{3}{*}{$E V(-1)$} & 156.9572 & -2.842947 & 1.140532 & 0.040745 & 0.138069 \\
\hline & $(37.9172)$ & $(1.687746)$ & $(0.44637)$ & $(0.08940)$ & $(0.04308)$ \\
\hline & [4.13948] & {$[-1.68475]$} & [2.55511] & [0.45578] & [3.20515] \\
\hline \multirow{3}{*}{$\mathrm{EV}(-2)$} & -136.6774 & 1.983120 & 0.001640 & -0.046786 & 0.079004 \\
\hline & $(29.1837)$ & $(1.29878)$ & $(0.34356)$ & $(0.06881)$ & $(0.03316)$ \\
\hline & {$[-4.68336]$} & {$[1.52690]$} & {$[0.00477]$} & {$[-0.67998]$} & {$[-2.38286]$} \\
\hline \multirow{3}{*}{ GINI(-1) } & -590.7491 & 3.093047 & 9.176323 & -0.109126 & -0.891256 \\
\hline & $(253.863)$ & $(11.2979)$ & $(2.98855)$ & $(0.59852)$ & $(0.28841)$ \\
\hline & [2.32704] & [0.27377] & [3.07050] & {$[-0.18233]$} & [3.09024] \\
\hline \multirow{3}{*}{ GINI(-2) } & -746.9026 & 31.01717 & -0.254608 & -0.21165 & -0.240249 \\
\hline & $(268.000)$ & $(11.9270)$ & (3.15497) & $(0.63185)$ & $(0.30447)$ \\
\hline & {$[-2.78695]$} & [2.60058] & {$[-0.08070]$} & {$[-0.33436]$} & {$[-0.78907]$} \\
\hline \multirow{3}{*}{$\mathrm{IDH}(-1)$} & -1310.214 & 1.120101 & -7.593937 & 0.496170 & -1.069158 \\
\hline & $(424.071)$ & $(18.8728)$ & $(4.99229)$ & $(0.99982)$ & $(0.48178)$ \\
\hline & {$[-3.08961]$} & {$[0.05935]$} & {$[-1.52113]$} & {$[0.49626]$} & {$[-2.21918]$} \\
\hline \multirow{3}{*}{ IDH(-2) } & 1076.978 & 31.72854 & -5.436621 & -0.571180 & -0.223288 \\
\hline & $(292.707)$ & $(13.0266)$ & (3.44583) & $(0.69011)$ & $(0.33254)$ \\
\hline & [3.67937] & [2.43568] & {$[-1.57774]$} & {$[-0.82767]$} & {$[-0.67146]$} \\
\hline \multirow{3}{*}{ C } & -1481.705 & 29.98606 & -2.724637 & 1.035264 & -3.145237 \\
\hline & (913.172) & $(40.6397)$ & $(10.7501)$ & $(2.15296)$ & $(1.03744)$ \\
\hline & {$[-1.62259]$} & {$[0.73785]$} & {$[-0.25345]$} & {$[0.48086]$} & {$[-3.03172]$} \\
\hline R-squared & 0.927212 & 0.997892 & 0.999898 & 0.963706 & 0.995179 \\
\hline Adj. R-squared & 0.823230 & 0.994881 & 0.999752 & 0.911858 & 0.988293 \\
\hline Sum sq. resids & 71.24676 & 0.141111 & 0.009874 & 0.000396 & $9.20 \mathrm{e}-05$ \\
\hline S.E. equation & 3.190315 & 0.141981 & 0.037557 & 0.007522 & 0.003624 \\
\hline F-statistic & 8.917010 & 331.4200 & 6853.024 & 18.58714 & 144.5105 \\
\hline Log likelihood & -37.92289 & 18.09635 & 42.03322 & 70.97860 & 84.12011 \\
\hline Akaike AIC & 5.435877 & -0.788484 & -3.448135 & -6.664289 & -8.124457 \\
\hline Schwarz SC & 5.979993 & -0.244368 & -2.904019 & -6.120173 & -7.580341 \\
\hline Mean dependent & 4.503500 & 14.02612 & 68.25164 & 0.526976 & 0.717344 \\
\hline S.D. dependent & 7.588031 & 1.984513 & 2.384699 & 0.025335 & 0.033498 \\
\hline
\end{tabular}


Cuenca, María Esperanza: Influencia del capital humano en las diferencias regionales en Colombia...

LÍNEA DE INVESTIGACIÓN: DESARROLLO INDUSTRIAL Y EMPRESARIAL

Tabla 5.

\begin{tabular}{|c|c|c|c|c|c|}
\hline & ANAL & GINI & EV & IDH & CRE \\
\hline \multirow{3}{*}{ ANAL(-1) } & 0.643944 & -0.005743 & -0.316064 & 0.005923 & -5.958412 \\
\hline & $(0.31906)$ & $(0.0 .2321)$ & $(0.08842)$ & $(0.01488)$ & $(12.2397)$ \\
\hline & [2.01826] & {$[-0.24738]$} & {$[-3.57458]$} & {$[0.39797]$} & {$[-0.48681]$} \\
\hline \multirow{3}{*}{ ANAL(-2) } & 2.301660 & 0.005205 & -0.303044 & 0.000887 & -18.35929 \\
\hline & $(0.70408)$ & $(0.05123)$ & $(0.19512)$ & $(0.03284)$ & $(27.0100)$ \\
\hline & [3.26902] & {$[0.10161]$} & {$[-1.55311]$} & {$[0.02700]$} & {$[-0.67972]$} \\
\hline \multirow{3}{*}{ GINI(-1) } & 45.27711 & -0.720054 & -5.422559 & 0.030385 & -437.3690 \\
\hline & $(11.6546)$ & $(0.84792)$ & (3.22981) & $(0.54362)$ & (447.093) \\
\hline & [3.88491] & {$[-0.84920]$} & {$[-1.67891]$} & {$[0.05589]$} & {$[-0.97825]$} \\
\hline \multirow{3}{*}{ GINI(-2) } & 29.36943 & -0.307607 & -0.866950 & -0.410250 & -448.2177 \\
\hline & $(9.74606)$ & $(0.70907)$ & $(2.70090)$ & $(0.45459)$ & (373.878) \\
\hline & [3.01347] & {$[-0.43382]$} & {$[-0.32099]$} & {$[-0.90245]$} & {$[-1.19883]$} \\
\hline \multirow{3}{*}{$E V(-1)$} & 10.67471 & -0.024284 & -0.884974 & -0.040547 & -57.10736 \\
\hline & (3.06992) & $(0.22335)$ & $(0.85076)$ & $(0.14319)$ & (117.768) \\
\hline & [3.47719] & {$[-0.10873]$} & {$[-1.04022]$} & {$[-0.28316]$} & {$[-0.48491]$} \\
\hline \multirow{3}{*}{$E V(-2)$} & -4.923773 & -0.026001 & 0.456673 & 0.050204 & -11.24160 \\
\hline & $(0.74880)$ & $(0.05448)$ & $(0.20751)$ & $(0.03493)$ & (28.7252) \\
\hline & {$[-6.57559]$} & {$[-0.47728]$} & [2.20071] & {$[1.43741]$} & {$[-0.39135]$} \\
\hline \multirow{3}{*}{$\mathrm{IDH}(-1)$} & -18.47134 & 0.744936 & -1.311595 & 1.639589 & -41.11979 \\
\hline & $(8.90684)$ & $(0.64801)$ & $(2.46833)$ & $(0.41545)$ & (341.684) \\
\hline & {$[-2.073384]$} & [1.14957] & {$[-0.53137]$} & [3.94654] & {$[-0.12034]$} \\
\hline \multirow{3}{*}{$\mathrm{IDH}(-2)$} & 5.703391 & -0.427654 & 4.651788 & -0.967790 & 145.3978 \\
\hline & $(14.3504)$ & $(1.04406)$ & $(3.97688)$ & $(0.66936)$ & (550.509) \\
\hline & {$[0.39744]$} & {$[-0.40961]$} & [1.16971] & {$[-1.144585]$} & {$[0.26412]$} \\
\hline \multirow{3}{*}{ CRE(-1) } & -0.006268 & 0.000271 & 0.001669 & 0.000480 & -0.119911 \\
\hline & $(0.00918)$ & $(0.00067)$ & $(0.00254)$ & $(0.00043)$ & $(0.35202)$ \\
\hline & {$[-0.68308]$} & {$[0.40518]$} & {$[0.65643]$} & [1.12198] & {$[-0.34064]$} \\
\hline \multirow{3}{*}{ CRE(-2) } & -0.020585 & -0.000570 & 0.003336 & 0.000408 & 0.319891 \\
\hline & $(0.01366)$ & $(0.00099)$ & $(0.00379)$ & $(0.00064)$ & $(0.52419)$ \\
\hline & {$[-1.50651]$} & {$[-0.57321]$} & {$[0.88086]$} & {$[0.64034]$} & {$[0.61026]$} \\
\hline \multirow{3}{*}{ C } & -454.9927 & 4.354928 & 107.5316 & -0.286705 & 5433.153 \\
\hline & (193.155) & $(14.0529)$ & $(53.5286)$ & $(9.00952)$ & (7409.82) \\
\hline & {$[-2.35558]$} & [0.30989] & [2.00886] & {$[-0.03182]$} & {$[0.73324]$} \\
\hline R-squared & 0.999511 & 0.980420 & 0.999852 & 0.987360 & 0.589790 \\
\hline Adj. R-squared & 0.998812 & 0.952449 & 0.999641 & 0.969304 & 0.003775 \\
\hline Sum sq. resids & 0.050163 & 0.000266 & 0.003852 & 0.000109 & 73.82120 \\
\hline S.E. equation & 0.084653 & 0.006159 & 0.023460 & 0.003949 & 3.247443 \\
\hline F-statistic & 1430.315 & 35.05107 & 4739.032 & 54.68164 & 1.006441 \\
\hline Log likelihood & 27.40482 & 74.57680 & 50.50384 & 82.57866 & -38.24236 \\
\hline Akaike AIC & -1.822758 & -7.064089 & -4.389316 & -7.953185 & 5.471374 \\
\hline Schwarz SC & -1.278642 & -6.519973 & -3.845200 & -7.409069 & 6.015490 \\
\hline Mean dependent & 9.172722 & 0.522944 & 70.42583 & 0.752833 & 3.8722 .78 \\
\hline S.D. dependent & 2.456058 & 0.028244 & 1.238718 & 0.022537 & 3.263590 \\
\hline
\end{tabular}


Tabla 6.

\begin{tabular}{|c|c|c|c|c|c|}
\hline & ANAL & CRE & EV & GINI & IDH \\
\hline \multirow{3}{*}{ ANAL(-1) } & 0.082592 & -6.915385 & 0.235228 & 0.001217 & -0.008155 \\
\hline & $(0.23552)$ & $(4.09480)$ & $(0.13705)$ & $(0.00658)$ & $(0.00473)$ \\
\hline & {$[0.35068]$} & {$[-1.68882]$} & [1.71633] & {$[0.18490]$} & {$[-1.72555]$} \\
\hline \multirow{3}{*}{ ANAL(-2) } & -0.752490 & 5.571744 & 0.310524 & 0.019273 & 0.005334 \\
\hline & $(0.21442)$ & (3.72798) & $(0.12478)$ & $(0.00599)$ & $(0.00430)$ \\
\hline & {$[-3.50948]$} & {$[1.49457]$} & [2.48866] & {$[-3.21634]$} & {$[1.23977]$} \\
\hline \multirow{3}{*}{ CRE $(-1)$} & -0.013700 & 0.899958 & -0.008947 & 0.000154 & -0.000132 \\
\hline & $(0.01918)$ & $(0.33352)$ & $(0.01116)$ & $(0.00054)$ & $(0.00038)$ \\
\hline & {$[-0.71418]$} & [2.69839] & {$[-0.80151]$} & [0.28710] & {$[-0.34163]$} \\
\hline \multirow{3}{*}{ CRE(-2) } & -0.014089 & -1.345381 & 0.021495 & -0.000222 & 0.000914 \\
\hline & $(0.03660)$ & $(0.63630)$ & $(0.02130)$ & $(0.00102)$ & $(0.00073)$ \\
\hline & {$[-0.38497]$} & {$[-2.11438]$} & [1.00928] & {$[-0.21666]$} & [1.22424] \\
\hline \multirow{3}{*}{$E V(-1)$} & 0.097906 & -16.49529 & 0.193269 & -0.011221 & -0.007739 \\
\hline & $(0.46741)$ & $(8.12658)$ & $(0.27200)$ & $(0.01306)$ & $(0.00938)$ \\
\hline & [0.20946] & {$[-2.02979]$} & {$[0.71056]$} & {$[-0.85903]$} & {$[-0.82510]$} \\
\hline \multirow{3}{*}{$\mathrm{EV}(-2)$} & -0.849689 & -1.427506 & 0.329238 & -0.038645 & 0.010223 \\
\hline & $(0.24631)$ & $(4.28245)$ & $(0.14333)$ & $(0.00688)$ & $(0.00494)$ \\
\hline & {$[-3.44967]$} & {$[-0.33334]$} & {$[2.29700]$} & {$[-5.61426]$} & {$[2.06836]$} \\
\hline \multirow{3}{*}{ GINI(-1) } & 0.830763 & -155.6952 & -8.029446 & -0.284147 & 0.421255 \\
\hline & $(6.30393)$ & $(109.603)$ & (3.66841) & $(0.17617)$ & $(0.12650)$ \\
\hline & {$[0.13178]$} & {$[-1.42054]$} & {$[-2.18881]$} & {$[-1.61293]$} & [3.33006] \\
\hline \multirow{3}{*}{ GINI(-2) } & 4.303580 & -356.0236 & -16.05457 & -0.073385 & -0.453856 \\
\hline & $(7.30628)$ & $(127.030)$ & $(4.25171)$ & $(0.20418)$ & $(0.14661)$ \\
\hline & {$[0.58902]$} & {$[-2.80267]$} & {$[-3.77603]$} & {$[-0.35941]$} & {$[-3.09557]$} \\
\hline \multirow{3}{*}{$\mathrm{IDH}(-1)$} & -18.45306 & 463.0850 & 14.45326 & 0.246363 & 0.991029 \\
\hline & $(13.0161)$ & $(226.304)$ & $(7.57441)$ & $(0.36375)$ & $(0.26119)$ \\
\hline & {$[-1.41771]$} & [2.04629] & {$[1.90817]$} & {$[0.67729]$} & [3.79423] \\
\hline \multirow{3}{*}{ IDH(-2) } & -1.549236 & -156.1505 & -1.957818 & -0.523442 & -0.623621 \\
\hline & $(6.53759)$ & $(113.665)$ & (3.80439) & $(0.18270)$ & $(0.13119)$ \\
\hline & {$[-0.23697]$} & {$[-1.37377]$} & {$[-0.51462]$} & {$[-2.86506]$} & {$[-4.75359]$} \\
\hline \multirow{3}{*}{ C } & 79.08985 & 1315.426 & 32.66366 & 4.560726 & 0.346347 \\
\hline & $(29.0712)$ & $(505.445)$ & $(16.9173)$ & $(0.81242)$ & $(0.58337)$ \\
\hline & [2.72055] & [2.60251] & [1.93079] & {$[5.61376]$} & {$[0.59370]$} \\
\hline R-squared & 0.995366 & 0.725257 & 0.999349 & 0.998387 & 0.992187 \\
\hline Adj. R-squared & 0.988746 & 0.332768 & 0.998419 & 0.996082 & 0.981026 \\
\hline Sum sq. resids & 0.099909 & 30.20123 & 0.033833 & 7.80E-05 & 4.02E-05 \\
\hline S.E. equation & 0.119468 & 2.077128 & 0.069522 & 0.003339 & 0.002397 \\
\hline F-statistic & 150.3618 & 1.847839 & 1074.515 & 433.2379 & 88.89849 \\
\hline Log likelihood & 21.20394 & -30.19849 & 30.94940 & 85.59871 & 91.56021 \\
\hline Akaike AIC & -1.133771 & 4.577610 & -2.216600 & -8.288745 & -8.951135 \\
\hline Schwarz SC & -0.589655 & 5.121726 & -1.672484 & -7.744629 & -8.407019 \\
\hline Mean dependent & 7.811279 & 3.656320 & 71.32720 & 0.494905 & 0.759718 \\
\hline S.D. dependent & 1.126174 & 2.542874 & 1.748407 & 0.053341 & 0.017404 \\
\hline
\end{tabular}


Cuenca, María Esperanza: Influencia del capital humano en las diferencias regionales en Colombia...

LÍNEA DE INVESTIGACIÓN: DESARROLLO INDUSTRIAL Y EMPRESARIAL

\section{Impulso - respuesta}

\section{Gráfico 1. Impulso Respuesta Región Elite I.}

Response to Choles ky One S.D. Innovations
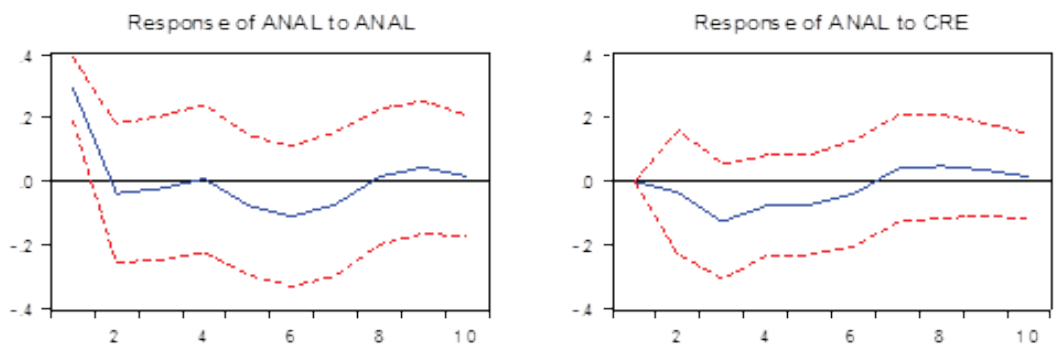

Res pons e of ANAL to EV
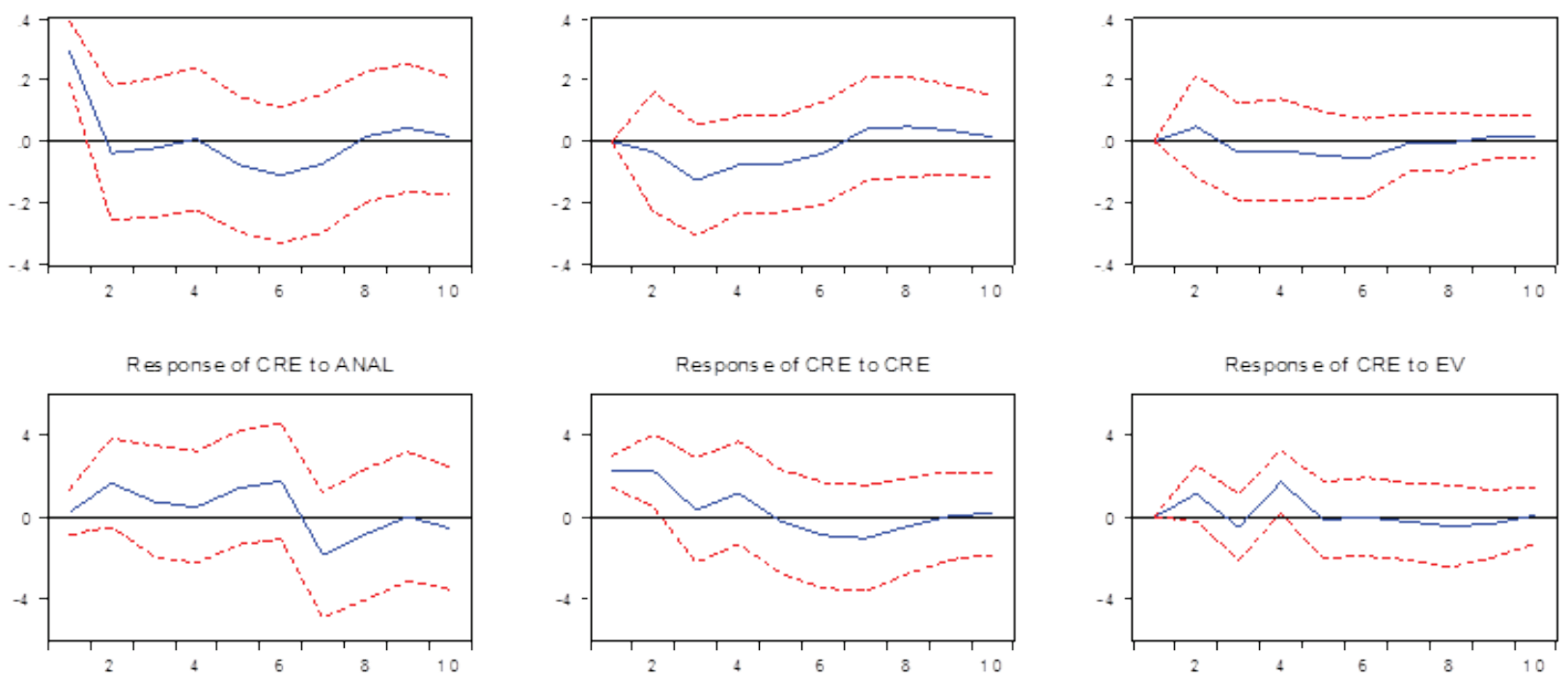

Response of EV to ANAL
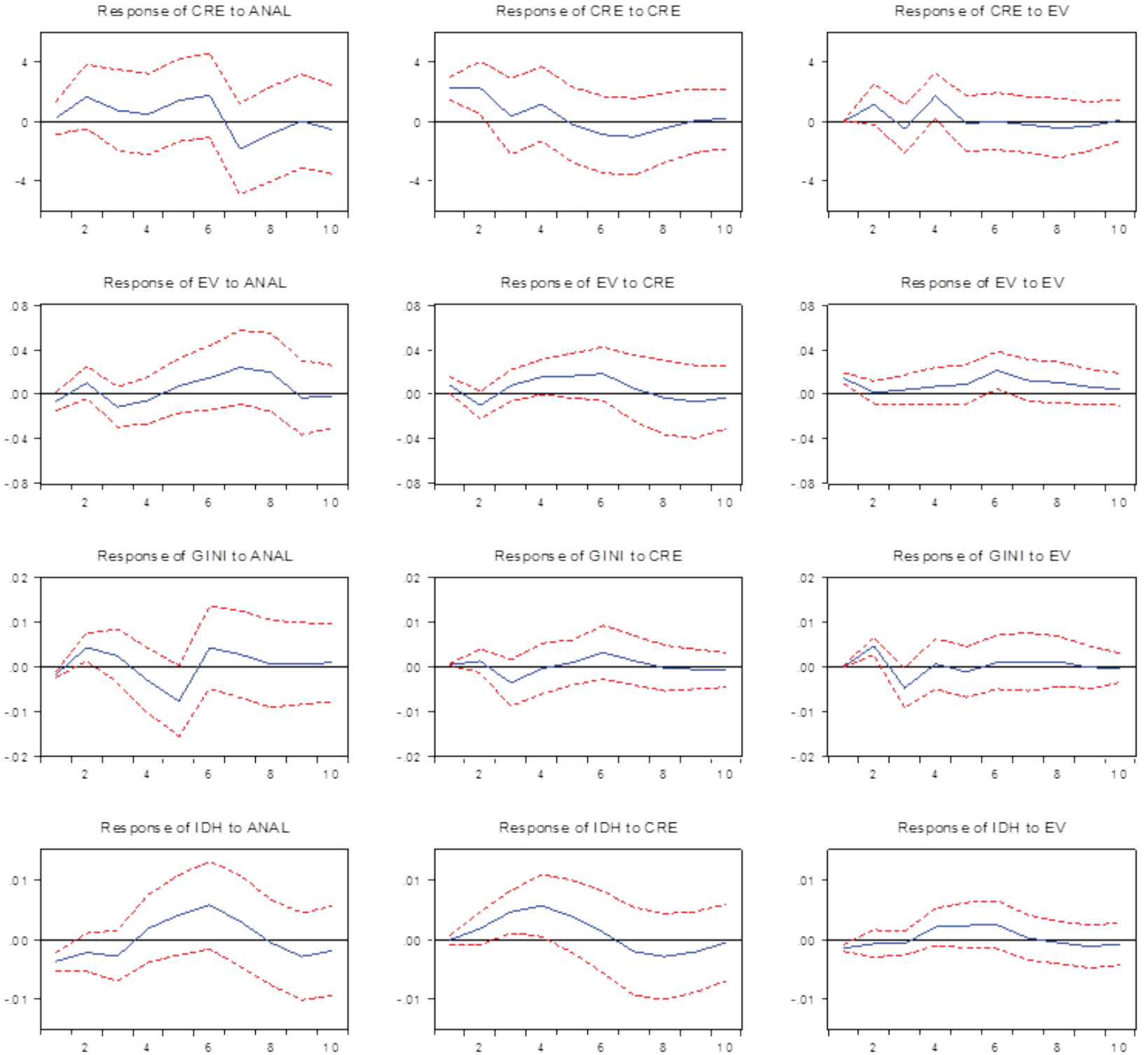

Fuente: Autor. Con base en Eviews 6.0 


\section{Gráfico 2. Impulso Respuesta Región Elite II.}

2S.E.
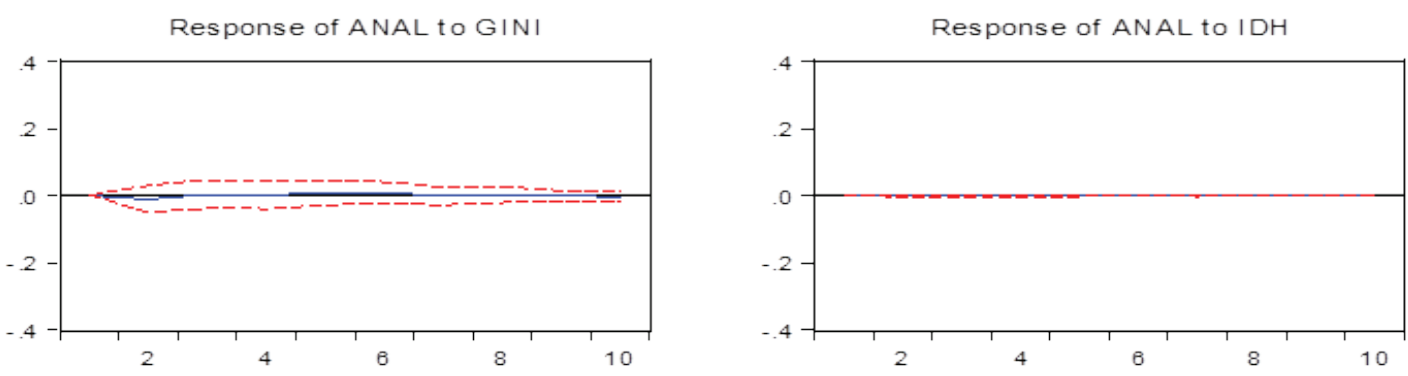

Response of CRE to GINI

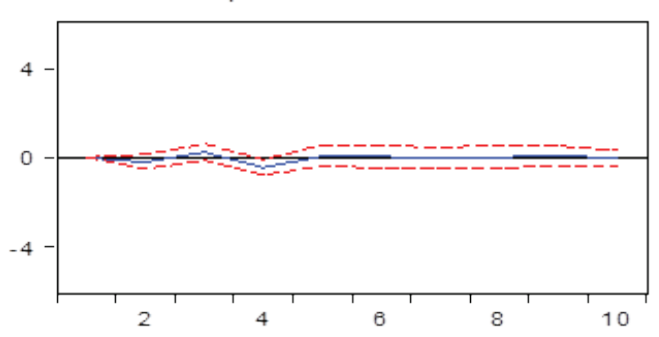

Response of CRE to IDH
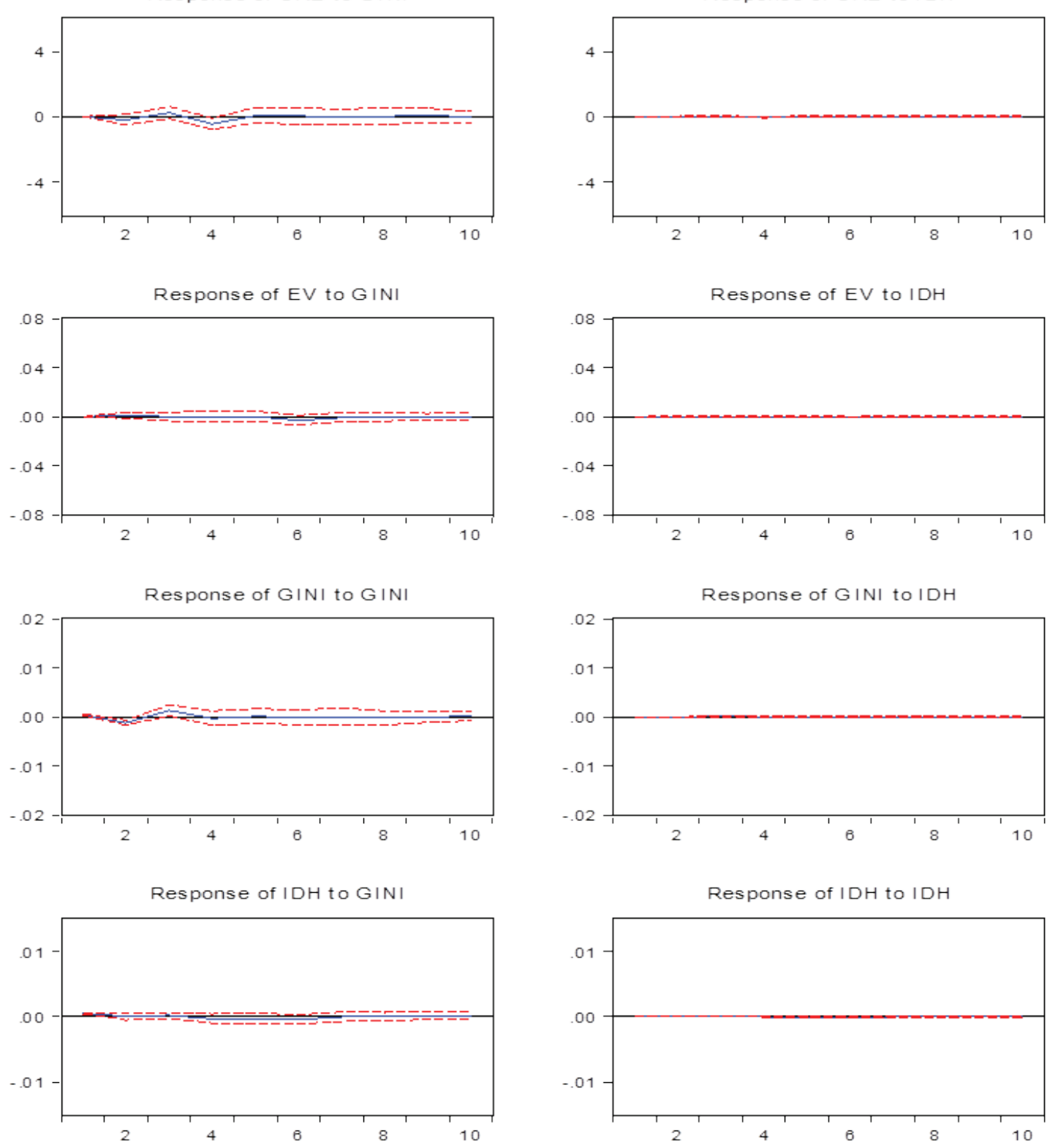

Fuente: Autor. Con base en Eviews 6.0 
Cuenca, María Esperanza: Influencia del capital humano en las diferencias regionales en Colombia...

LÍNEA DE INVESTIGACIÓN: DESARROLLO INDUSTRIAL Y EMPRESARIAL

\section{Gráfico 3. Impulso Respuesta Región Norte.}

$\pm 2 S . E$
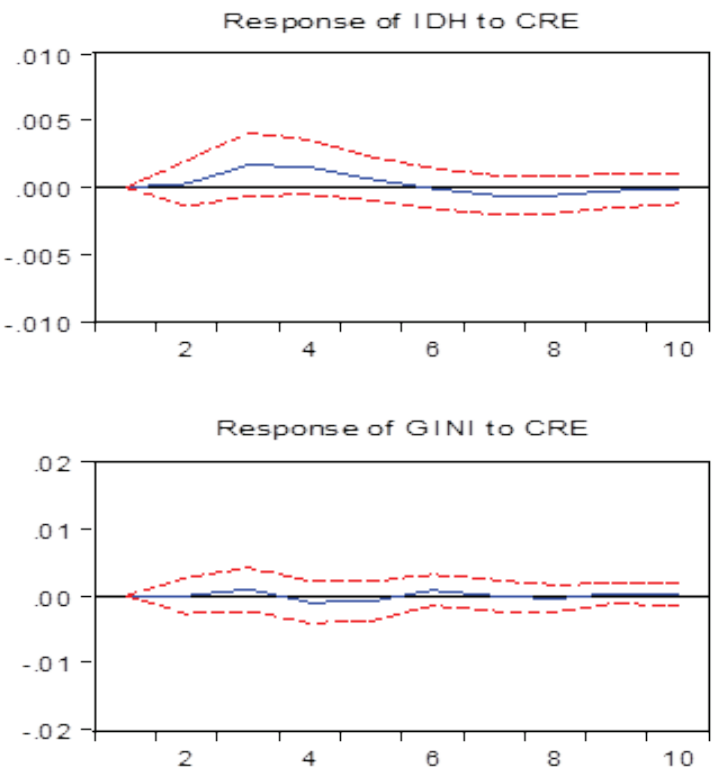

Response of EV to CRE

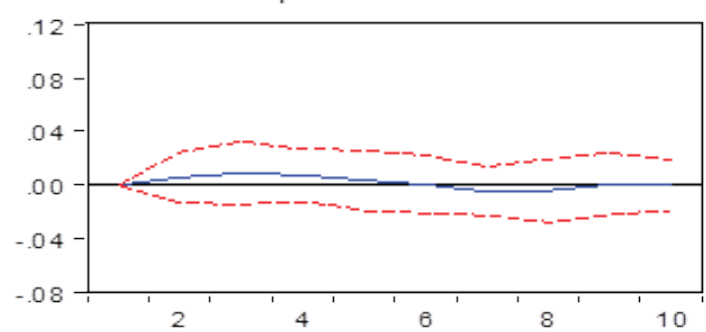

Response of CRE to CRE

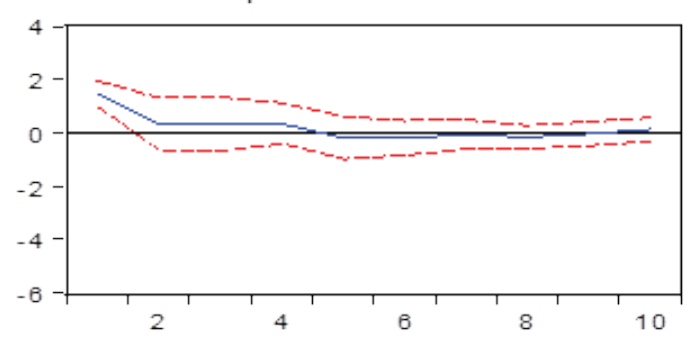

Response of ANAL to CRE

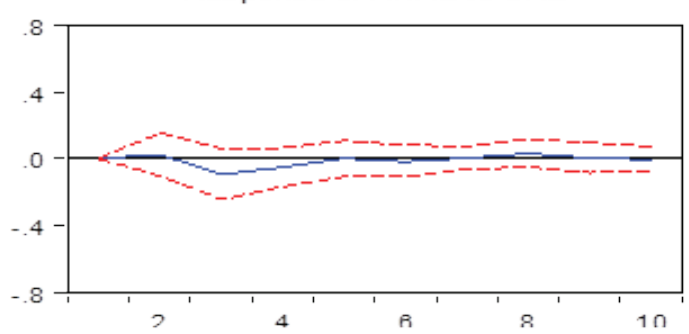

Response of IDH to ANAL

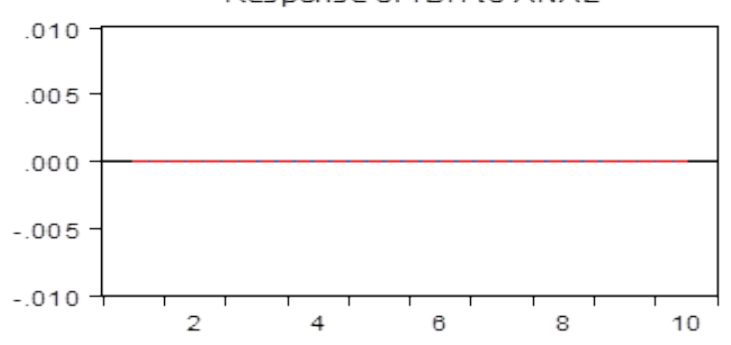

Response of GINI to ANAL

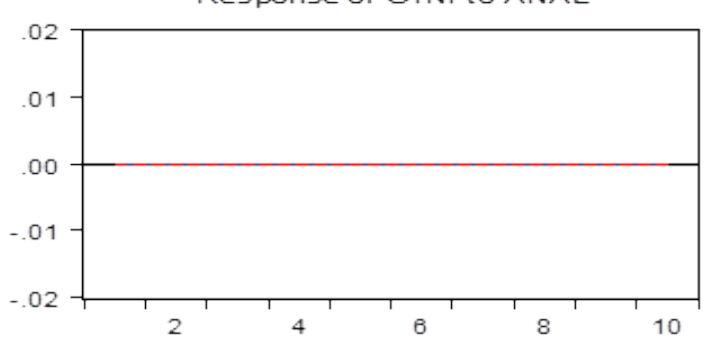

Response of EV to ANAL

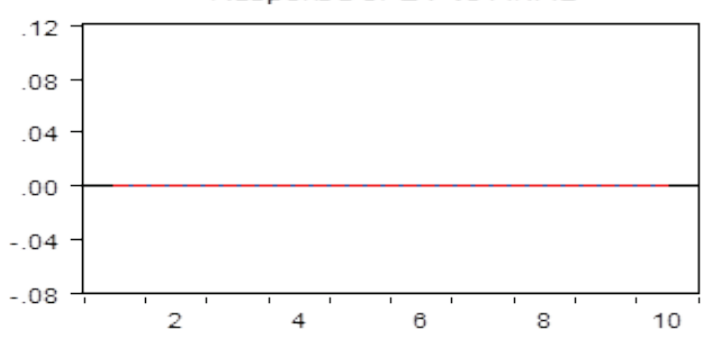

Response of CRE to ANAL

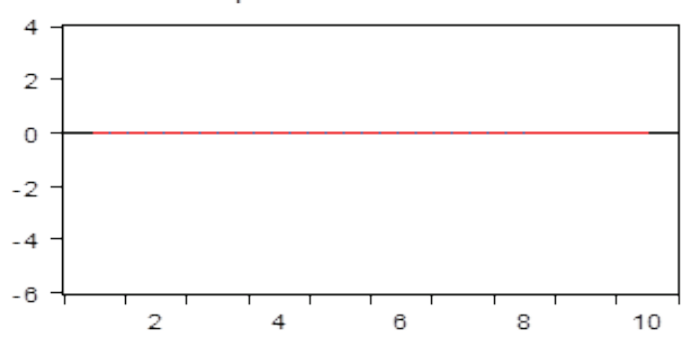

Response of ANAL to ANAL

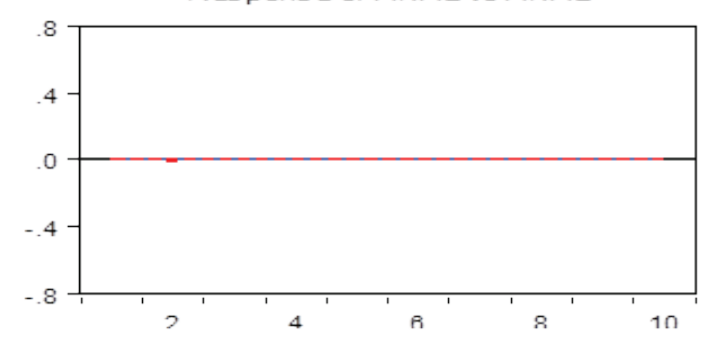

Fuente: Autor. Con base Eviews 6.0 


\section{Grafico 4. Impulso Respuesta Región Periférica.}
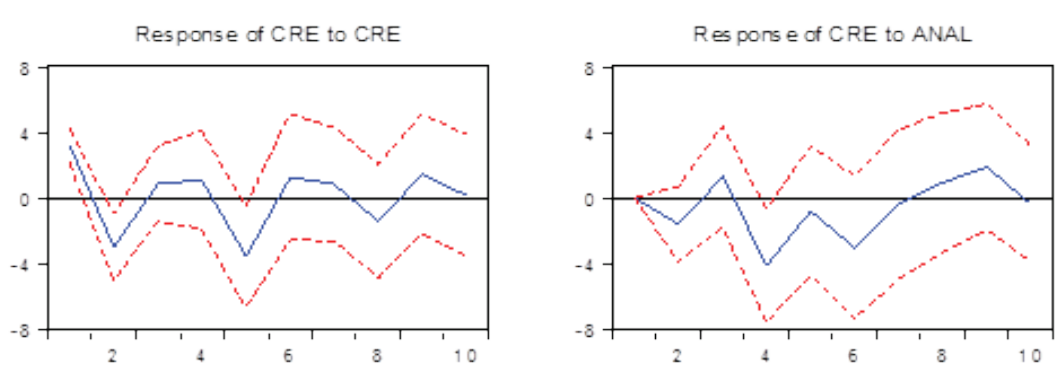

Response to Choles ky One S.D. Innovations I
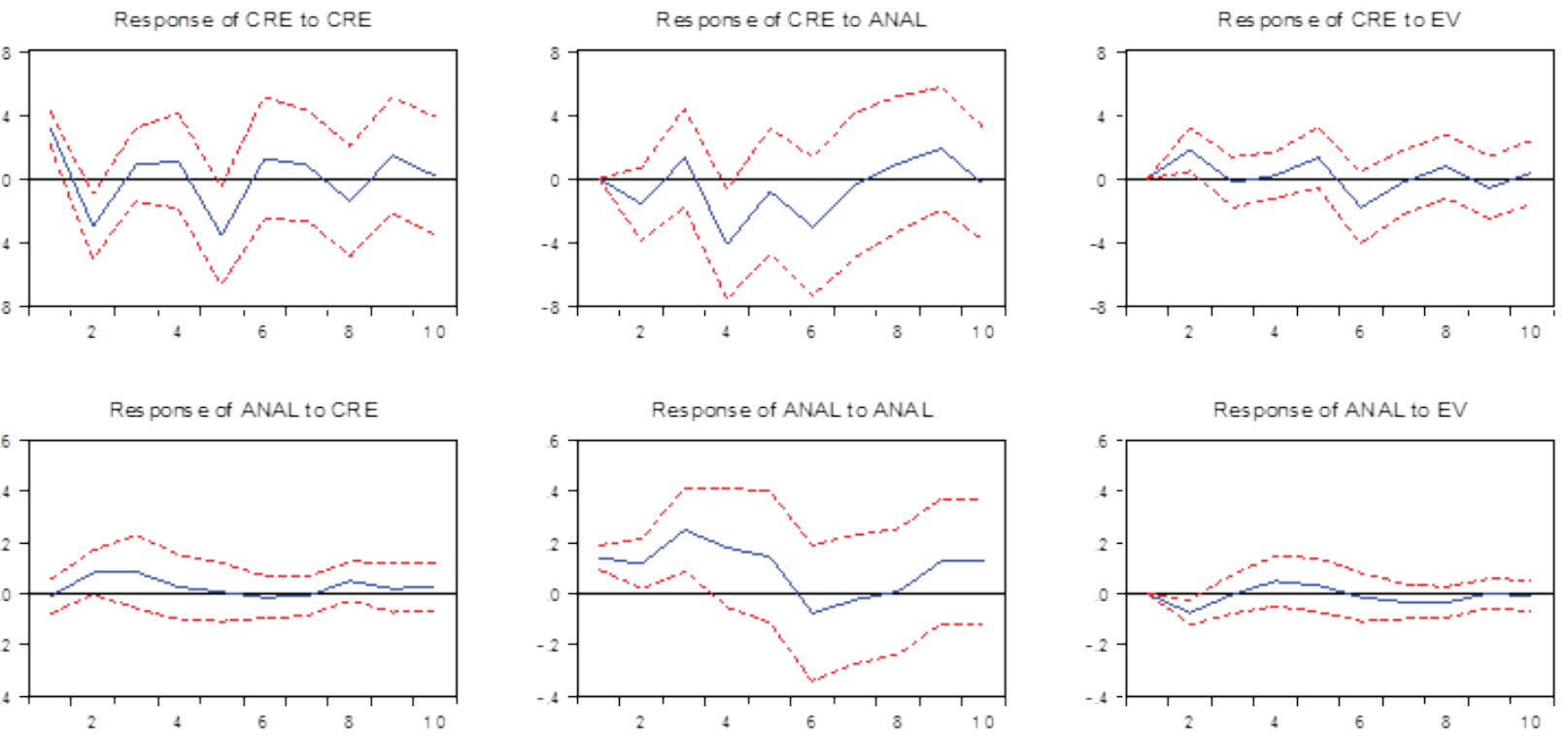

Respons e of EV to CRE

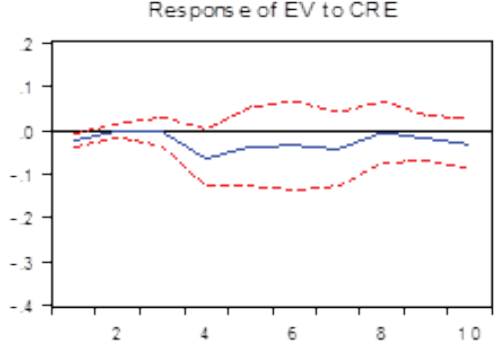

Response of EV to ANAL
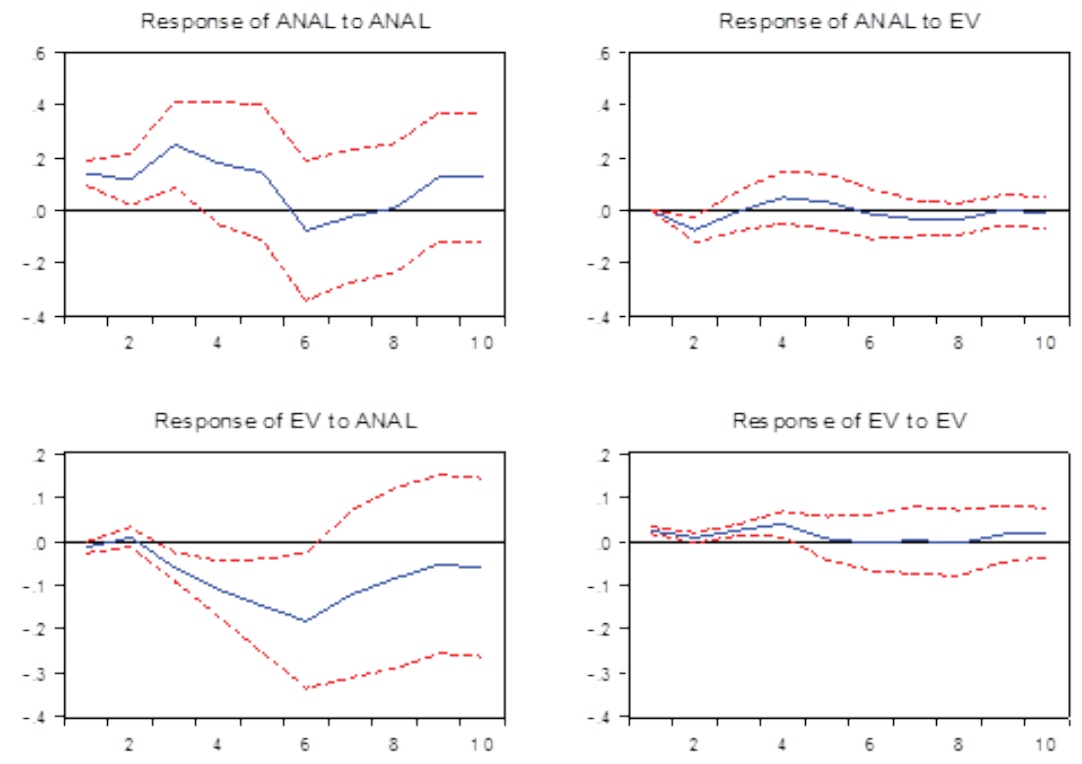

Res pons e of GINI to CRE
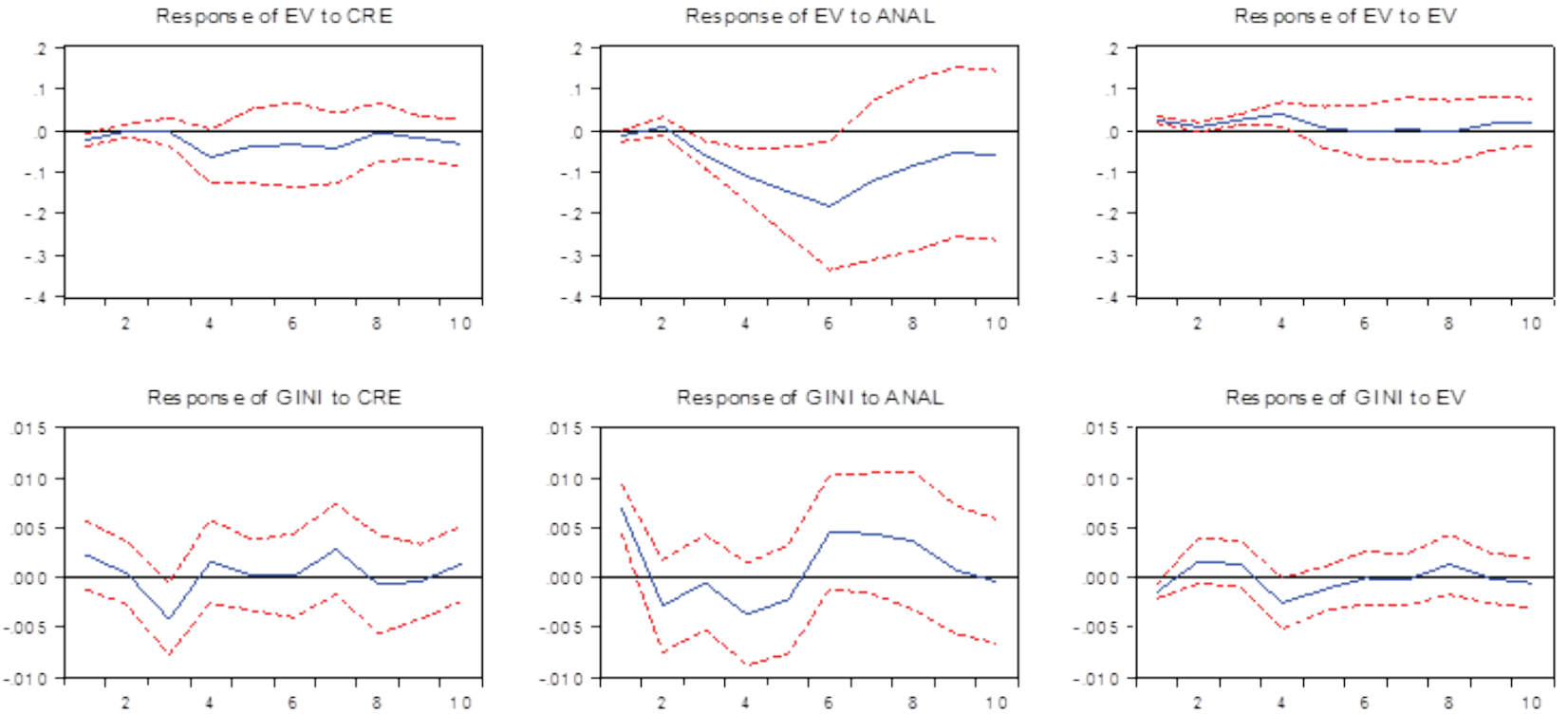

esponse of IDH to CRE
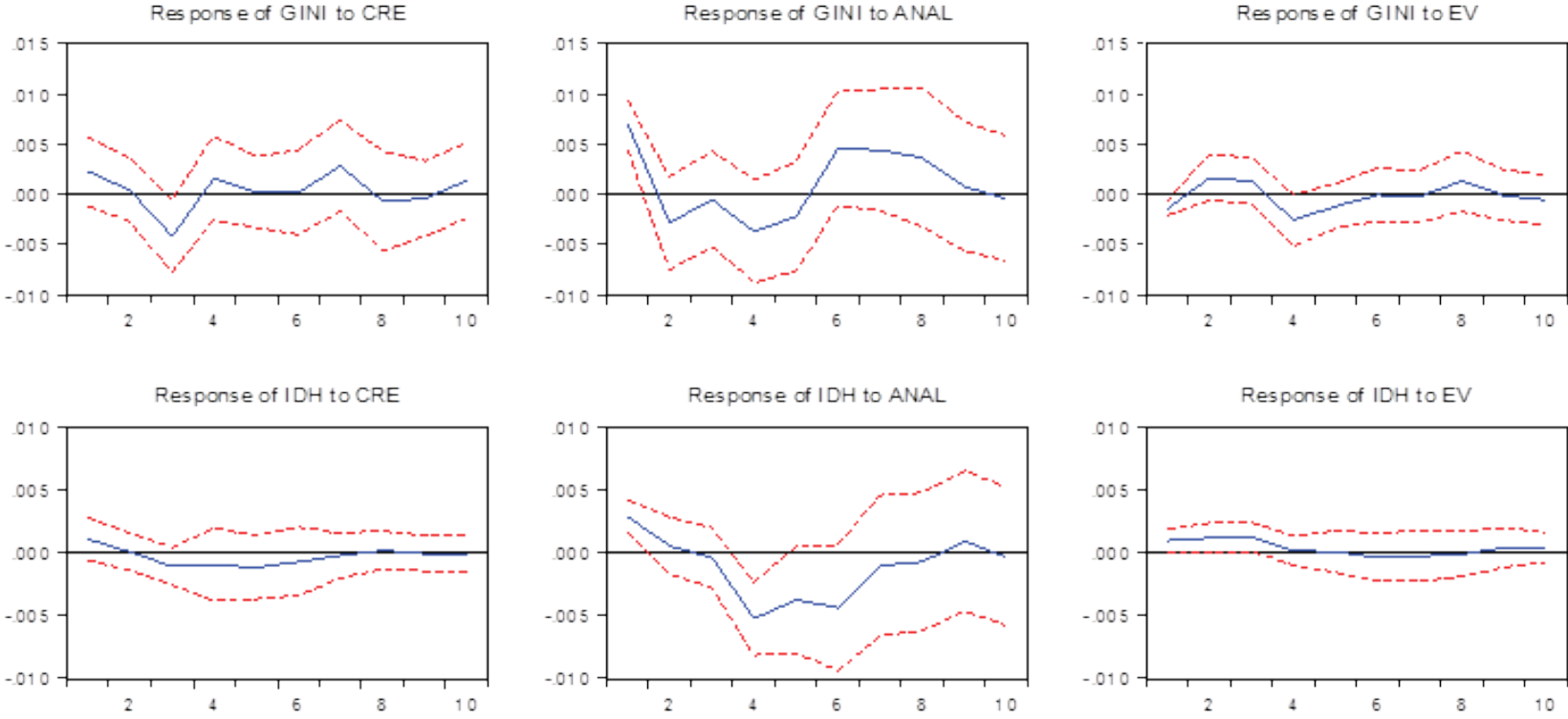

Fuente: Autor. Con base en Eviews 6.0 
Cuenca, María Esperanza: Influencia del capital humano en las diferencias regionales en Colombia...

\section{LÍNEA DE INVESTIGACIÓN: DESARROLLO INDUSTRIAL Y EMPRESARIAL}

Grafico 5. Impulso Respuesta Región Central I.
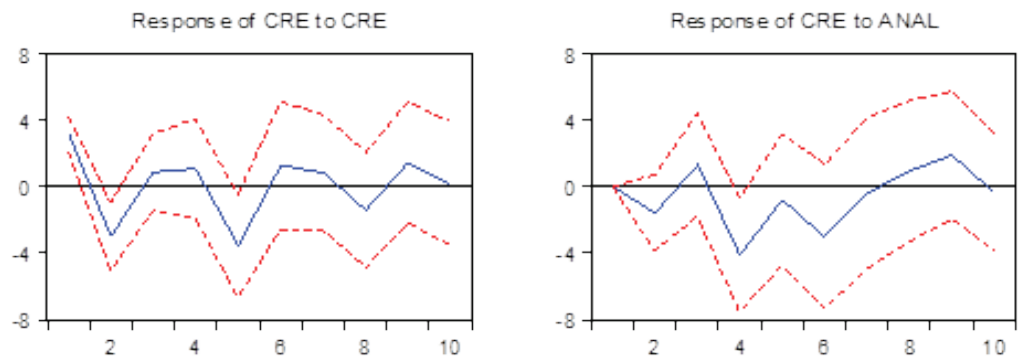

Response to Chol eskyOne S.D. Innovations \pm :
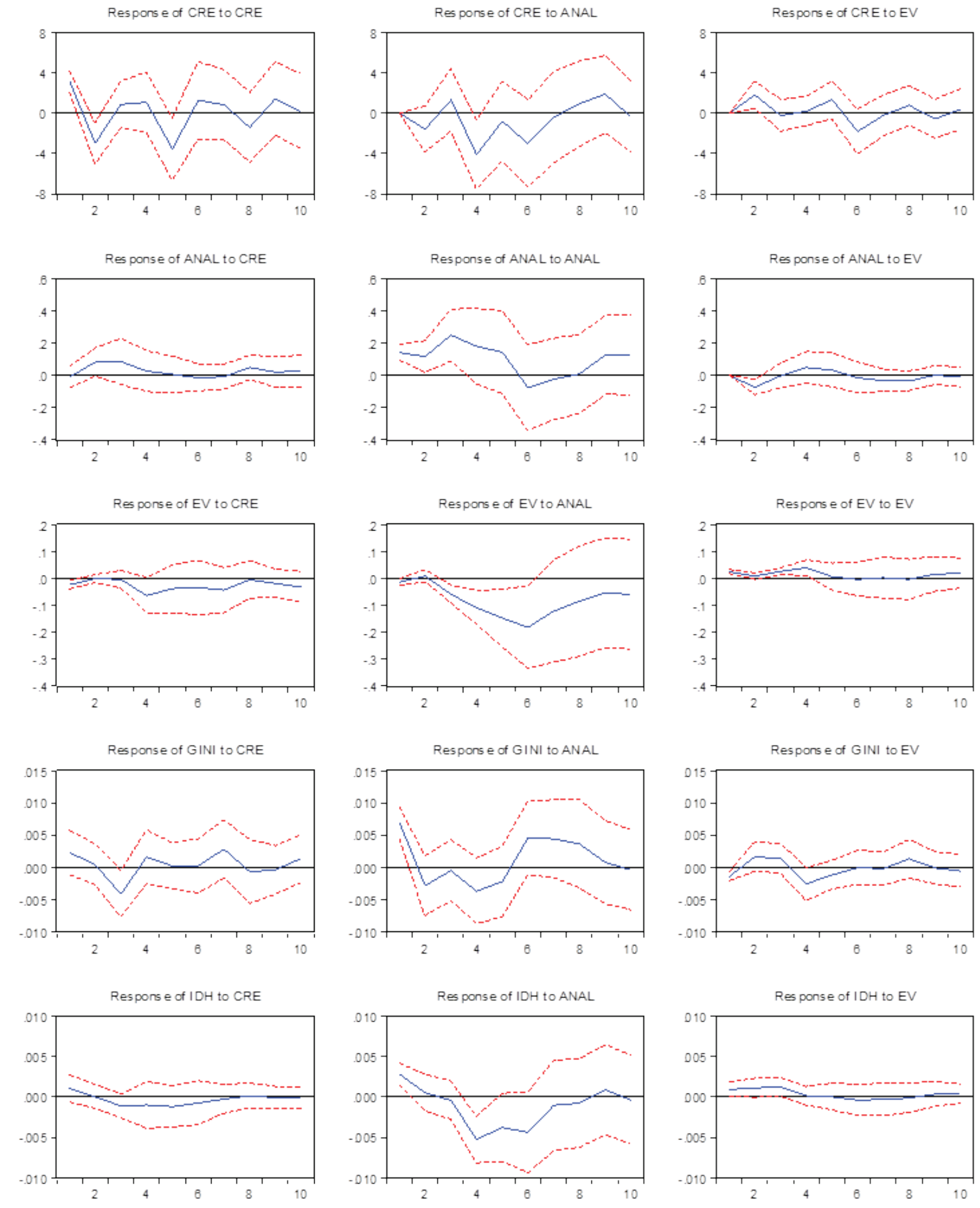

Fuente: Autor. Con base en Eviews 6.0 


\section{Grafico 6. Impulso Respuesta Región Central II.}
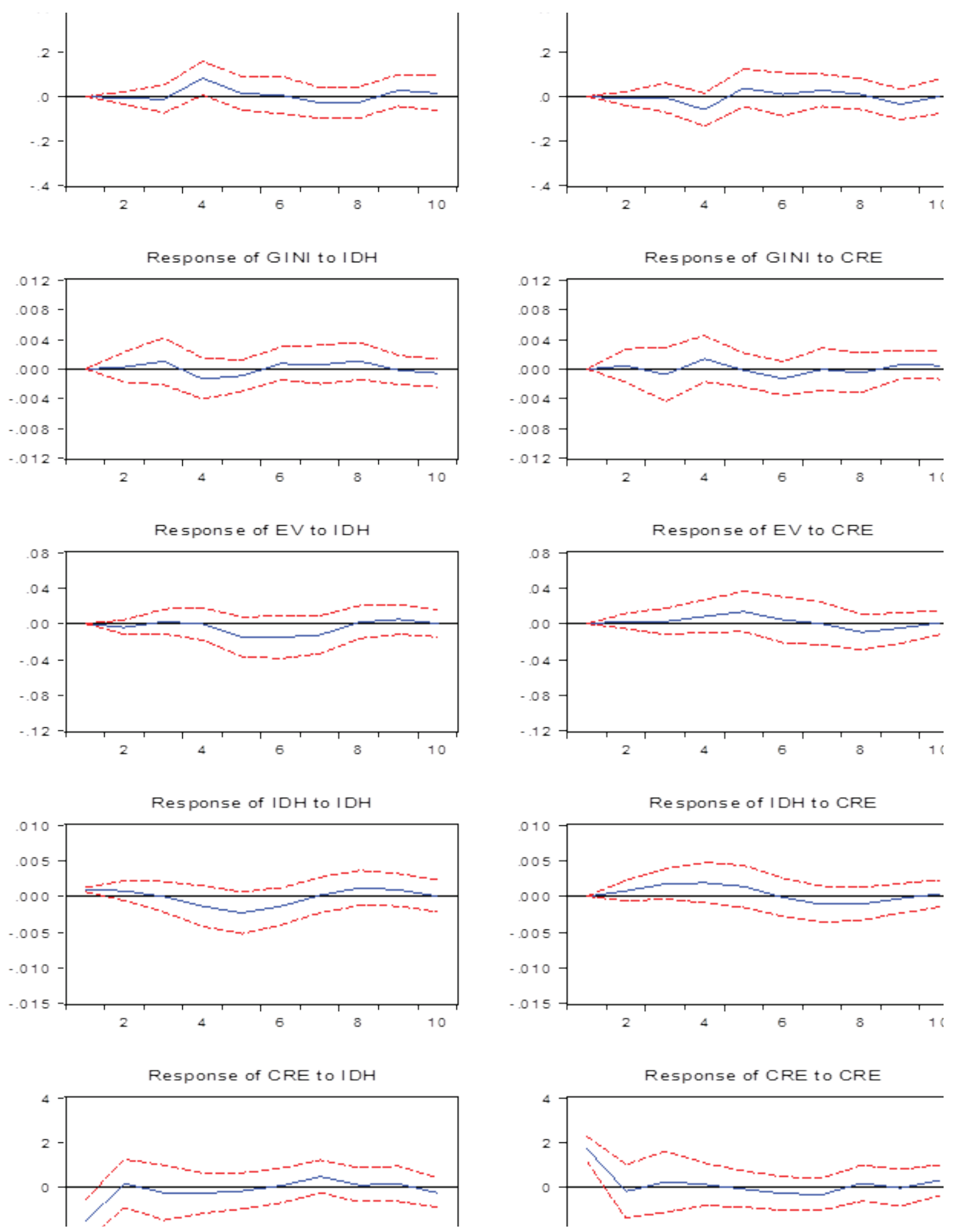

Fuente: Autor. Con base en Eviews 6.0 
Cuenca, María Esperanza: Influencia del capital humano en las diferencias regionales en Colombia...

\section{LÍNEA DE INVESTIGACIÓN: DESARROLLO INDUSTRIAL Y EMPRESARIAL}

\section{Grafico 7. Impulso Respuesta Región Cafetera.}

Res pons e to CholeskyOne S.D. Innovations

Response of ANAL to ANAL

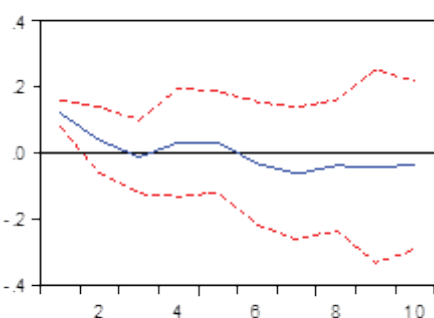

Response of CRE to ANAL

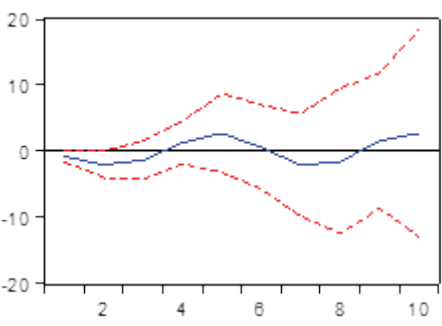

Response of EV to ANAL

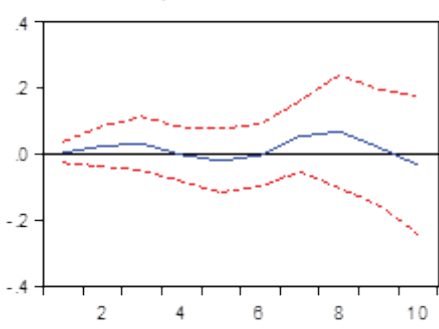

Response of GINI to ANAL

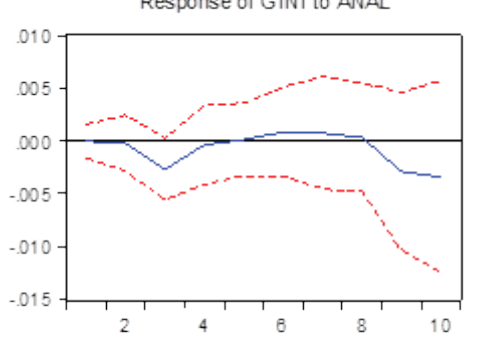

Response of IDH to ANAL

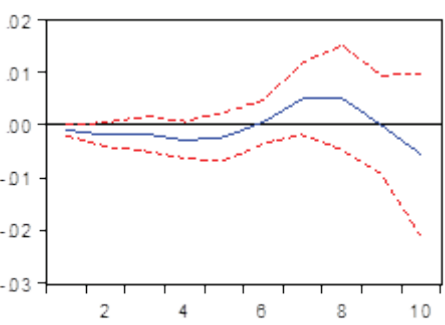

Response of ANAL to CRE

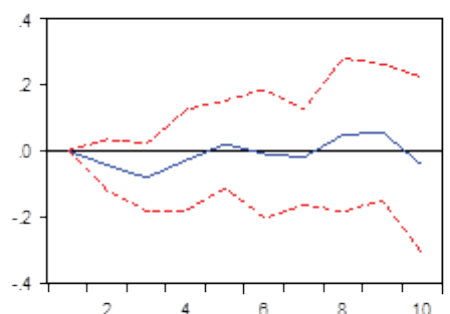

Response of CRE to CRE

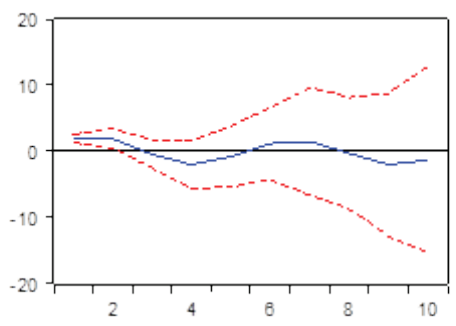

Response of EV to CRE

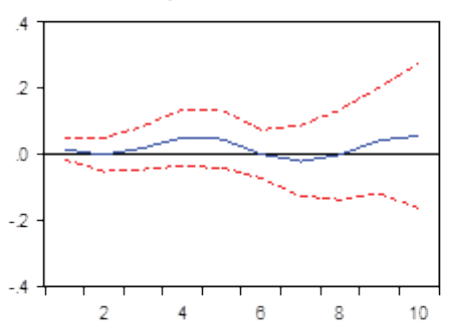

Response of GINI to CRE

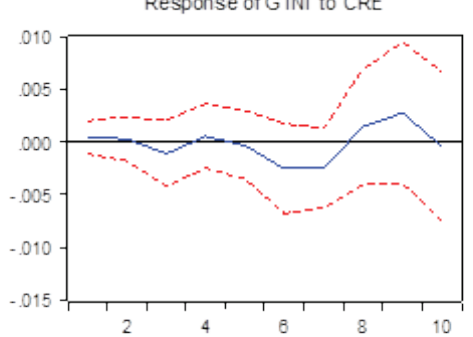

Response of IDH to CRE

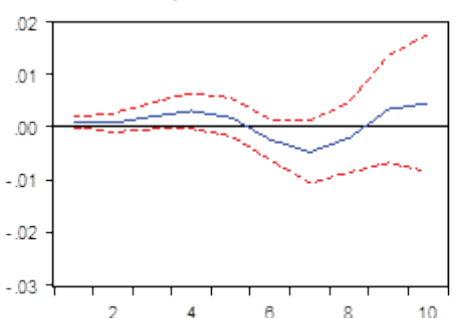

Response of ANAL to EV

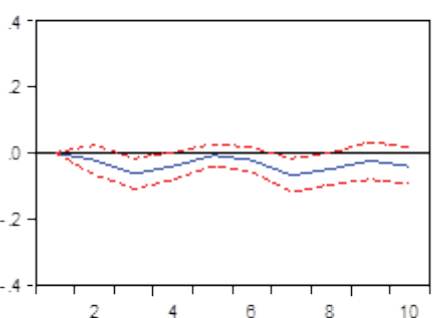

Response of CRE to EV

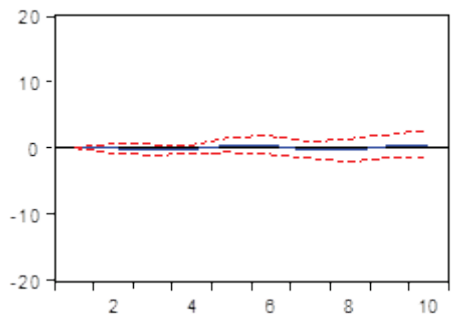

Response of EV to EV

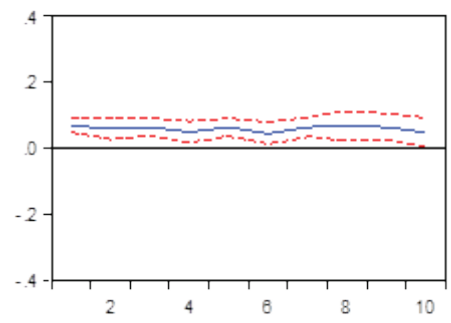

Response of GINI to EV

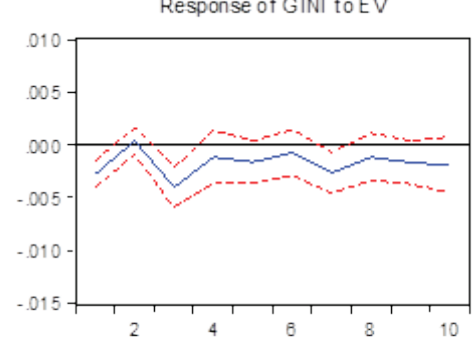

Response of IDH to EV

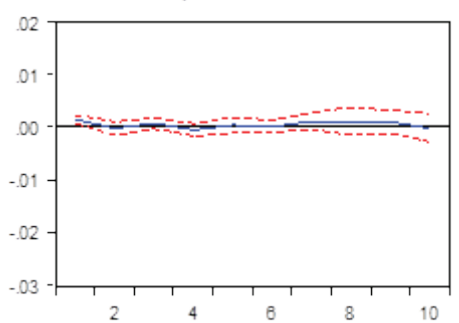

Fuente: Autor. Con base en Eviews 6.0 


\section{Grafico 8. Impulso respuesta Región Cafetera II.}

2S.E.

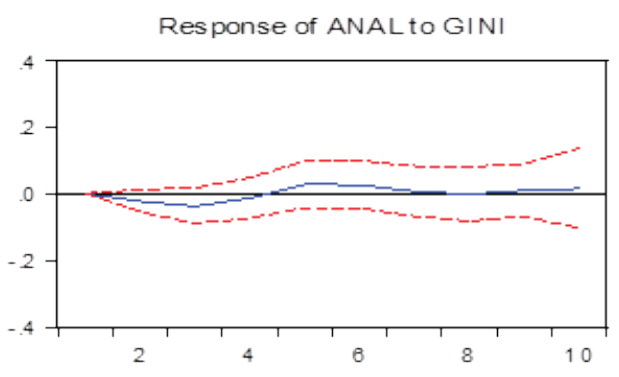

Response of CRE to GINI

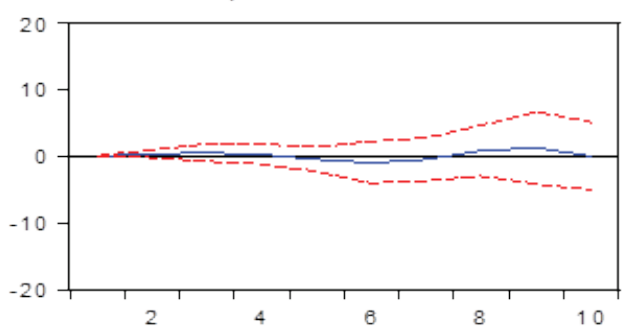

Response of EV to GINI

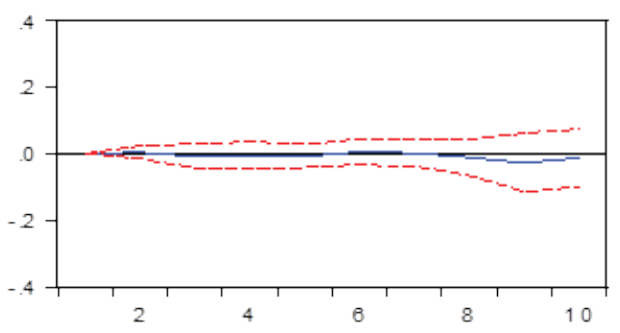

Res ponse of GINI to GINI

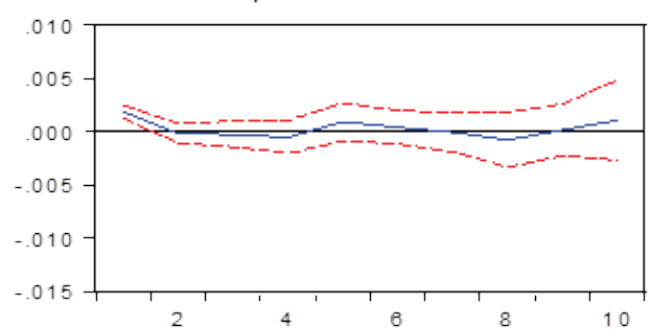

Response of IDH to GINI

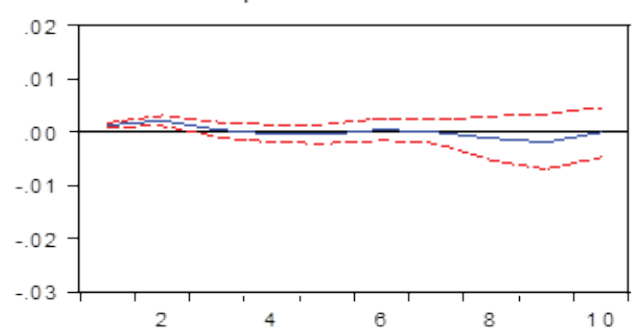

Response of ANAL to IDH

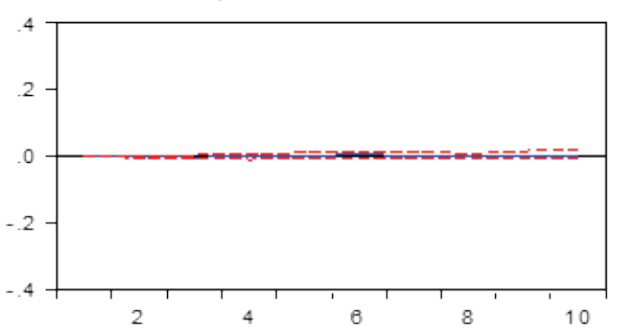

Response of CRE toIDH

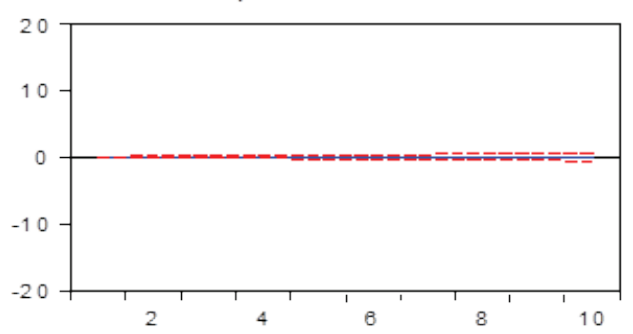

Response of EV to IDH

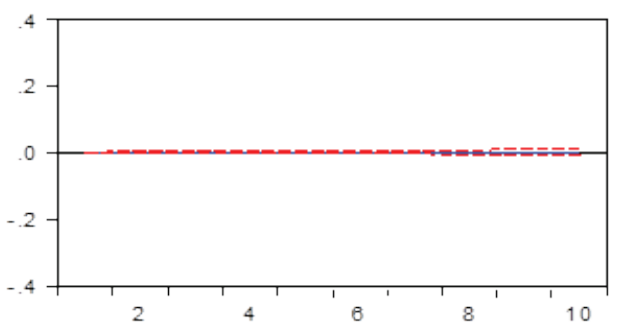

Response of GINI toIDH

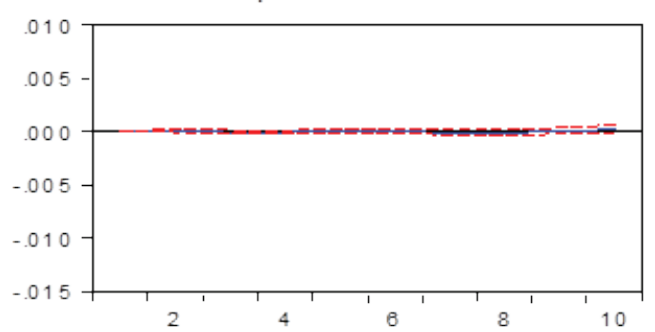

Response of IDH toIDH

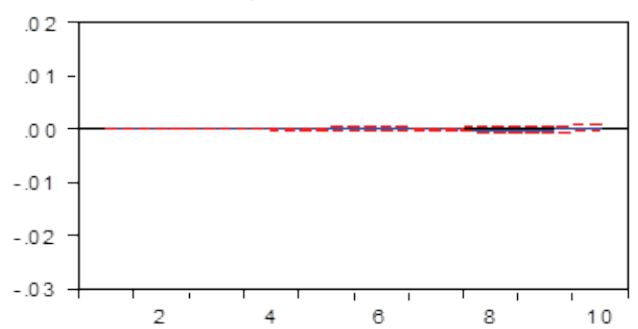

Fuente: Autor. Con base es Eviews 6.0 